\title{
Sparse spectral-tau method for the three-dimensional helically reduced wave equation on two-center domains
}

\author{
STEPHEN R. LAU* \\ Department of Mathematics and Statistics \\ University of New Mexico \\ Albuquerque, NM 87131. \\ RICHARD H. Price' \\ Center for Gravitational Wave Astronomy \\ Department of Physics and Astronomy \\ University of Texas at Brownsville \\ Brownsville, TX 78520
}

\begin{abstract}
We describe a multidomain spectral-tau method for solving the three-dimensional helically reduced wave equation on the type of two-center domain that arises when modeling compact binary objects in astrophysical applications. A global two-center domain may arise as the union of Cartesian blocks, cylindrical shells, and inner and outer spherical shells. For each such subdomain, our key objective is to realize certain (differential and multiplication) physical-space operators as matrices acting on the corresponding set of modal coefficients. We achieve sparse banded realizations through the integration "preconditioning" of Coutsias, Hagstrom, Hesthaven, and Torres. Since ours is the first three-dimensional multidomain implementation of the technique, we focus on the issue of convergence for the global solver, here the alternating Schwarz method accelerated by GMRES. Our methods may prove relevant for numerical solution of other mixed-type or elliptic problems, and in particular for the generation of initial data in general relativity.
\end{abstract}

*lau@math.unm.edu

$\dagger$ rprice@phys.utb.edu 


\section{INTRODUCTION AND PRELIMINARIES}

\section{A. Introduction}

This paper describes spectral methods designed with a specific application in mind: numerical solution of a mixed-type problem arising in gravitational physics. In reviewing an ongoing program to construct helically symmetric solutions to the Einstein equations, this introduction recalls the origins of this problem below. However, this paper also serves another purpose; it demonstrates that spectral-tau integration preconditioning 1 yields highly accurate numerical solutions to the helically reduced wave equation (HRWE), a mixed-type, variable coefficient, linear partial differential equation (PDE) problem, here posed on a nontrivial three-dimensional (3D) domain. Ref. [1] offered spectral-tau integration preconditioning as a general-purpose strategy for spectral approximation of differential equations, and that reference provides the most thorough description and analysis of the technique; related techniques were explored in [2] (integration postconditioning) and [3] (nodal integration preconditioning), with applications described in, for example, Refs. [4, 5]. However, heretofore, spectral-tau integration preconditioning has primarily been studied either in the ODE context or for PDE problems posed on single and basic two-dimensional (2D) domains (rectangles, annuli, and disks), although we have earlier studied a 2D multidomain scenario [6] as a warm-up to this work. While the current paper only considers the HRWE, a challenging model problem for the aforementioned target application, it shows how to implement the technique in a 3D multidomain setting, addressing several key conditioning issues which would seem to generically arise in higher dimensional settings. Therefore, our work should facilitate the use of spectral-tau integration preconditioning for other elliptic or mixed-type PDE problems. We provide more context and a fuller description of these issues below, but now turn to the physical problem which has motivated our work.

The advent of gravitational wave detection has driven theoretical studies of gravitational wave sources. A source that is possibly interesting for ground-based detectors, and perhaps the most exciting source for space-based detectors, is the inspiral of two comparable mass black holes and their merger to form a single black hole. The early stage of inspiral is modeled with reasonable accuracy by perturbations of the Newtonian analysis, and the post-merger stage can be analyzed with black hole perturbation theory. The most difficult stage to analyze is the intermediate stage, when a few orbits remain. This epoch of inspiral is too late for a modified Newtonian approach, but too early for black hole perturbation theory. Yet this is the epoch in which a large part of the gravitational wave energy is generated.

The importance of, and difficulty in, analyzing this epoch was the original motivation for an innovative approximation, the periodic standing wave (PSW) method. This approach uses the fully nonlinear field interactions, but models the binary compact objects to be forever on circular orbits of constant radius. Therefore, both the compact source motions and the fields exhibit helical symmetry. Not only does this symmetry reduce the number of independent variables, it also completely changes the nature of the governing PDEs, turning the problem from the hyperbolic evolution of initial data to one of mixed-type that is elliptic near a rotation axis and hyperbolic well outside the axis and beyond the orbits in the wave zone of the system. More details of this astrophysical background are given in [6]. Here we only point out that recent supercomputer evolutions of initial black hole binary data have

\footnotetext{
1 We use this term to refer to a specific technique reviewed below; however, insofar as our work is concerned the word preconditioning might be a misnomer. In any case, the technique does achieve sparsification, and this is the aspect of the technique we focus on here.
} 
been run stably for many orbits in the intermediate epoch. See, for example, Refs. [7 23$]$ (not an exhaustive list), and 24] for a recent review. Even the inspiral of binaries with large mass ratios [21, 22] or high spins [23], both particularly challenging cases, can now be computed. To be sure, recent successes with purely hyperbolic numerical evolutions have undercut the original motivation for the PSW approximation. Nevertheless, there remains a niche for the PSW approximation for the following reasons. First, it should provide a test bench for understanding nonlinear gravitational radiation reaction as a local process. Second, a helically symmetric solution of the Einstein equations would be, of its own accord, of bewitching interest.

The numerical computation of PSW fields has, in fact, already been carried out, using a single grid and a unique method devised especially for the problem by one of us (RHP) and coworkers. These computations were done in a series of steps [25 29] moving from linearized scalar fields up to and including the nonlinear tensor fields of general relativistic gravity. However, the method used proved inherently too limited in accuracy to be useful. Furthermore, despite the attractive simplicity of the computational method, its implementation for general relativistic tensor fields proved very challenging. The astrophysical PSW problem, therefore, can be viewed as not yet solved. The spectral methods described here are designed to solve this astrophysical problem to high accuracy. In any case, as mentioned above, our work is relevant as a successful use of spectral-tau integration preconditioning for the solution of PDEs (even of mixed-type) on nontrivial 3D domains. From this standpoint, the astrophysical problem simply provides a convenient application, with a particularly interesting feature. In the astrophysical problem, the region in which the PDEs are hyperbolic - the distant wave zone - is a region in which the PDEs have only very small nonlinearities. The strong linearities, near the source objects, are confined to a region in which the PDEs are elliptic. While we do not consider nonlinearities in the current paper, the methods we introduce for our linear model problem deliver sufficient accuracy that nonlinearities can almost surely be included.

Multidomain spectral methods for the binary inspiral of compact relativistic objects are not new. In pioneering work, nodal (i.e. collocation) methods were used by Pfeiffer et al. [30, 31] for the elliptical problem of constructing binary black hole initial data, and are now being used by the Caltech-Cornell-CITA collaboration (see, for example, [20]) in the fundamentally hyperbolic evolution problem. This work, now highly developed, relies on SpEC (the Spectral Einstein Code [32]), a large C++ project chiefly developed by Pfeiffer, Kidder, and Scheel, but also involving many other researchers and developers. One certainly might attack the problem we consider with that software; in particular with SpEC's EllipticModule which uses finite-difference preconditioning [33, 34] and is also already configured to solve nonlinear problems. Indeed, the EllipticModule has been used to solve the initial value constraint equations on essentially the same type of domain we consider below 2 , and we suspect that SpEC could be used to solve our problem to high accuracy. In any case, to date the 3D mixed problem considered here has not been numerically solved via spectral methods.

Our previous study [6] applied a modal multidomain spectral-tau method to a model nonlinear problem of two strong field sources in binary motion with only two spatial dimensions. That study also relied on integration preconditioning, although the relevant linear systems were inverted by direct rather than iterative methods (which was possible since the $2 \mathrm{D}$ problem was less memory intensive). Our 2D study, a proof of concept, showed that high accuracy could be achieved with relatively modest memory and run-time requirements.

\footnotetext{
${ }^{2}$ In fact, the domain decomposition of Pfeiffer et al. 30, 31] motivated our own choice.
} 


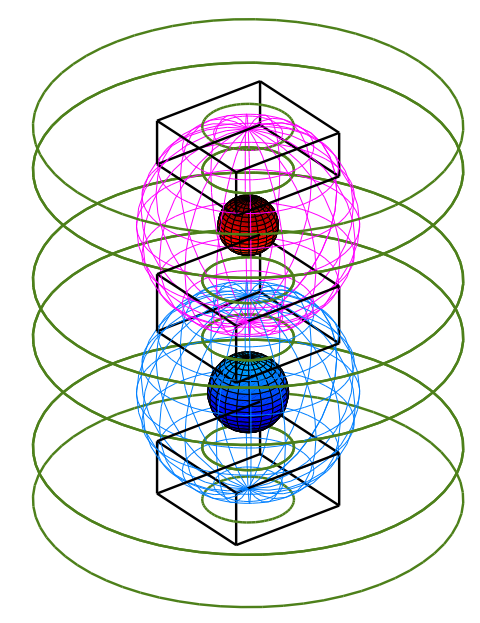

(a) 3d view of domain decomposition.

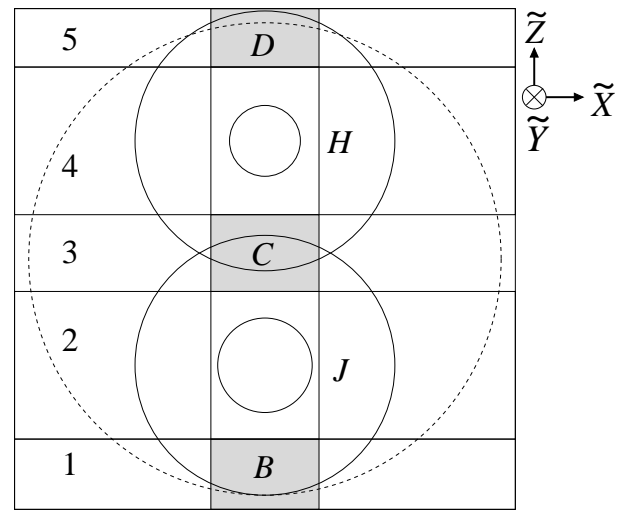

(b) Cross-sectional view.

FIG. 1. Domain Decomposition. The whole inner configuration of 10 subdomains is enclosed within an outer spherical shell which is not shown, save for its inner boundary in (b). Our total configuration is therefore comprised of 11 subdomains.

Here we generalize our 2D method to $3 \mathrm{D}$, that is to three spatial dimensions and one time dimension, reduced to a problem with three independent variables by the imposition of helical symmetry. Due to the larger set of modes needed for the 3D problem, iterative solution of the relevant linear system is now necessary. We use the generalized minimum residual method (GMRES) [35, 36]. Since the amount of work and storage per iteration increases with the iteration count [35, 36], preconditioning is a crucial issue (and here we mean further, one might even say genuine, preconditioning on top of the "integration preconditioning"). Through a multilevel preconditioning scheme, we will achieve near round-off accuracy for large truncations $(\simeq 600,000$ unknowns $)$ with modest iteration counts. Moreover, as we achieve a sparse formulation of the relevant linear system, each iteration is also fast.

\section{B. Specification of the problem}

Before writing down our mixed-type PDE problem, we describe the two-center (hereafter 2-center) domain $\mathcal{D}$ on which the problem is posed, first recalling the coordinate conventions of [26]. Let $\{x, y, z\}$ represent the inertial Cartesian system related to the spherical-polar system $\{r, \theta, \phi\}$ in the usual physicist's convention ( $\theta$ and $\phi$ are respectively the polar and azimuthal angles). We then introduce a "comoving" Cartesian system

$$
\widetilde{z}=r \cos \theta, \quad \widetilde{x}=r \sin \theta \cos (\phi-\Omega t), \quad \widetilde{y}=r \sin \theta \sin (\phi-\Omega t),
$$

where $\Omega<1$ is a fixed rotation rate. Note that the $\widetilde{z}$ and $z$-axes coincide, and both are the azimuthal axis. Via a simple permutation, we then define a new comoving system

$$
\widetilde{X}=\widetilde{y}, \quad \widetilde{Y}=\widetilde{z}, \quad \widetilde{Z}=\widetilde{x},
$$

for which the $\widetilde{Z}$-axis is not the azimuthal axis. If we imagine two compact objects with "centers" located at $\boldsymbol{\xi}_{1}(t)=a_{1} \cos (\Omega t) \mathbf{e}_{x}+a_{1} \sin (\Omega t) \mathbf{e}_{y}$ and $\boldsymbol{\xi}_{2}(t)=-a_{2} \cos (\Omega t) \mathbf{e}_{x}-a_{2} \sin (\Omega t) \mathbf{e}_{y}$ 


\begin{tabular}{|c|c|c|c|}
\hline \multicolumn{3}{|c|}{ Spherical shells } \\
\hline \hline$J$ & $0.4 \leq r \leq 1.1$ & $0 \leq \theta<2 \pi$ & $0 \leq \phi \leq \pi$ \\
$H$ & $0.3 \leq r \leq 1.1$ & $0 \leq \theta<2 \pi$ & $0 \leq \phi \leq \pi$ \\
$O$ & $2.0 \leq r \leq 150.0$ & $0 \leq \theta<2 \pi$ & $0 \leq \varphi \leq \pi$ \\
\hline \hline \multicolumn{3}{|c|}{ Cylindrical shells } \\
\hline 1 & $0.452 \leq \rho \leq 2.120$ & $0 \leq \phi<2 \pi$ & $-2.120 \leq \tilde{Z} \leq-1.525$ \\
2 & $0.452 \leq \rho \leq 2.120$ & $0 \leq \phi<2 \pi$ & $-1.525 \leq \tilde{Z} \leq-0.275$ \\
3 & $0.452 \leq \rho \leq 2.120$ & $0 \leq \phi<2 \pi$ & $-0.275 \leq \tilde{Z} \leq+0.375$ \\
4 & $0.452 \leq \rho \leq 2.120$ & $0 \leq \phi<2 \pi$ & $+0.375 \leq \tilde{Z} \leq+1.625$ \\
5 & $0.452 \leq \rho \leq 2.120$ & $0 \leq \phi<2 \pi$ & $+1.625 \leq \tilde{Z} \leq+2.120$ \\
\hline \hline \multicolumn{3}{|c|}{ Blocks } \\
\hline$B$ & $-0.640 \leq \tilde{X} \leq 0.640$ & $-0.640 \leq \tilde{Y} \leq 0.640$ & $-2.120 \leq \tilde{Z} \leq-1.525$ \\
$C$ & $-0.640 \leq \tilde{X} \leq 0.640$ & $-0.640 \leq \tilde{Y} \leq 0.640$ & $-0.275 \leq \tilde{Z} \leq+0.375$ \\
$D$ & $-0.640 \leq \tilde{X} \leq 0.640$ & $-0.640 \leq \tilde{Y} \leq 0.640$ & $+1.625 \leq \tilde{Z} \leq+2.120$ \\
\hline
\end{tabular}

TABLE I. Particular domain decomposition. The inner shells $J$ and $H$ are centered at $(\tilde{X}, \tilde{Y}, \tilde{Z})=$ $(0,0,-0.9)$ and $(\tilde{X}, \tilde{Y}, \tilde{Z})=(0,0,1.0)$, and for each shell the polar system $(r, \theta, \phi)$ is relative to the Cartesian system arising from translation of the $\{\tilde{X}, \tilde{Y}, \tilde{Z}\}$ system to the shell's corresponding origin. For each cylinder, the cylindrical system $(\rho, \phi, \tilde{Z})$ has the standard relationship with the $\{\tilde{X}, \tilde{Y}, \tilde{Z}\}$ Cartesian system. Finally, the outer shell is $O$ centered at the origin of the $\{\tilde{X}, \tilde{Y}, \tilde{Z}\}$ system, but now the $(r, \theta, \varphi)$ system is relative to the $\{\tilde{x}, \tilde{y}, \tilde{z}\}$ system.

in the inertial $\{x, y, z\}$ system, then the $\widetilde{Z}$-axis connects those compact objects. That is, $\boldsymbol{\xi}_{1}=a_{1} \mathbf{e}_{\widetilde{Z}}$ and $\boldsymbol{\xi}_{2}=-a_{2} \mathbf{e}_{\widetilde{Z}}$. We introduce the coordinates $\{\widetilde{r}, \widetilde{\theta}, \widetilde{\varphi}\}$ as spherical coordinates relative to the comoving $\{\widetilde{x}, \widetilde{y}, \widetilde{z}\}$ system. We will exclusively work with the comoving systems (or simple translations or polar versions thereof), but we will often suppress tildes when doing so will not cause confusion. We will, for example, use $\{r, \theta, \varphi\}$, hereafter, to mean $\{\widetilde{r}, \widetilde{\theta}, \widetilde{\varphi}\}$; these coordinates should not be confused with $\{r, \theta, \phi\}$ of Eq. (11).

Relative to the system $\{\tilde{X}, \tilde{Y}, \tilde{Z}\}$, the 2-center domain $\mathcal{D}$ that we have used is depicted in Fig. 1. Topologically, the domain $\mathcal{D}$ is a large solid 3D ball with two excised regions (each a smaller solid 3D ball). The global domain $\mathcal{D}$ has been broken into 11 subdomains, each sufficiently simple to allow for spectral expansions in terms of classical basis functions. A large outer shell (labeled $O$ for "out") is not shown in Fig. 1, but the remaining 10 subdomains which comprise the "inner region" are shown. The inner region is comprised of two "inner shells" (spherical shells labeled $J$ and $H$ ), three "blocks" (rectangular subdomains labeled $B, C$, and $D$ ), and five "cylinders" (cylindrical shells labeled 1, 2, 3, 4, and 5). Table【 lists the parameters which specify the subdomains comprising $\mathcal{D}$, along with the numerical values we have used in the computations to be reported below. Its caption describes the relationship between the parameters and the appropriate comoving system.

The HRWE problem we consider is as follows:

$$
L \psi=g \text { on } \mathcal{D}, \quad \psi=h^{-} \text {on } \partial H^{-} \cup \partial J^{-}, \quad\left(\frac{\partial}{\partial r}-\Omega \frac{\partial}{\partial \varphi}+\frac{1}{r}\right) \psi=h^{+} \text {on } \partial O^{+},
$$

where the defining operator is

$$
L=\frac{\partial^{2}}{\partial \tilde{x}^{2}}+\frac{\partial^{2}}{\partial \tilde{y}^{2}}+\frac{\partial^{2}}{\partial \tilde{x}^{2}}-\Omega^{2}\left(\tilde{x} \frac{\partial}{\partial \tilde{y}}-\tilde{y} \frac{\partial}{\partial \tilde{x}}\right)^{2}=\frac{\partial^{2}}{\partial \widetilde{X}^{2}}+\frac{\partial^{2}}{\partial \widetilde{Y}^{2}}+\frac{\partial^{2}}{\partial \widetilde{Z}^{2}}-\Omega^{2}\left(\widetilde{Z} \frac{\partial}{\partial \widetilde{X}}-\tilde{X} \frac{\partial}{\partial \widetilde{Z}}\right)^{2} .
$$


Here the constant $\Omega$ is the rotation rate, and $g$ is a prescribed source. As described in, for example, [6] this problem arises via a helically reduction of the inhomogeneous $3+1$ wave equation (see also the Appendix). Notice that the problem includes Dirichlet conditions set on the inner boundaries of the spherical shells $J$ and $H$. The boundary condition set on the outer boundary of the spherical shell $O$ is of radiative type, and is here expressed in terms of the polar coordinates $\{r, \theta, \varphi\}$ relative to $\{\tilde{x}, \tilde{y}, \tilde{z}\}$. Although this radiation condition is described precisely below, it may here be thought of as an inhomogeneous Sommerfeld condition. (The inhomogeneity $h^{+}$in (3) is a nonlocal expression.) This paper will consider only the variable-coefficient linear problem (3). For numerical tests, $g$ is taken as zero on $\mathcal{D}$, but with distributional support, point sources, located at the centers, $\boldsymbol{\xi}_{1}$ and $\boldsymbol{\xi}_{2}$, of $J$ and $H$. For this choice of $g$, an exact solution is described in the Appendix. While we only consider the linear scalar problem (3), the helical reduction of the Einstein equations described in [27, 28] involves a tensorial field resolved into ten coupled "helical scalars" $\psi^{(\alpha \beta)}$ each of which obeys a copy $L \psi^{(\alpha \beta)}=g^{(\alpha \beta)}$ of the above equation. However, for this formulation $g^{(\alpha \beta)}$ is now not an external source, but rather is a nonlinear coupling function of the helical scalars built with lower-order terms including some first derivatives. Therefore, clearly solving the linear problem that we consider is the first step towards considering the helically reduced Einstein equations.

\section{Overview of 3D spectral-tau integration "preconditioning"}

Mostly focusing on the 3D HWRE in three Cartesian variables on a rectangular block, this subsection gives a short overview of integration preconditioning for spectral-tau methods, in particular focusing on the Kronecker product representations necessary for 3D. We hope that this overview will provide the reader with enough context to follow the heavy details encountered later when applying the technique on 3D spherical and cylindrical shells. Our earlier paper [6] gave a fuller account of essentially the same issues for 2D, many of which change little in going to 3D. Therefore, in order to here avoid a prohibitively long discussion, we have opted for a short overview, and one tied to our particular problem, pointing the reader to [6] for more details.

The following overview makes use of matrices $D^{k}$ and $B_{[n]}^{m}$. These respectively represent $k$ th order differentiation and $m$ th order integration with respect to a basis of Chebyshev polynomials. As explained later, the subscript $[n]$ indicates that the first $n$ rows of a matrix are empty. We do not here provide precise expressions for $D^{k}$ and $B_{[n]}^{m}$; however, we list the following key properties exploited later: (i) $D^{k}$ is dense upper triangular, (ii) $B_{[n]}^{n}$ is sparse and banded with upper and lower bandwidth $n$, and (iii) $B_{[n]}^{n} D^{k}=B_{[n]}^{n-k}$ for $n \geq k$. Here $B_{[n]}^{0} \equiv I_{[n]}$ is the identity matrix, except that each entry in its first $n$ rows is a 0 . Our earlier paper [6] gave the precise expressions for $D \equiv D^{1}, D^{2}, B_{[1]} \equiv B_{[1]}^{1}$, and $B_{[2]}^{2}$; our treatment of the 3D HRWE (a second order equation) only requires these matrices. That reference also discusses the necessary rescalings of these matrices for work with an arbitrary interval rather than the standard interval $[-1,1]$ for Chebyshev polynomials. Of course, Ref. [1] also considered such expressions and identities, even for more general basis functions. 


\section{Direct product representations}

A function on any of our 3D subdomains is encoded by the modal coefficients for its spectral expansion, and this set of modal coefficients is often here viewed as a direct (Kronecker) product. For example, let us consider a rectangular block delineated by the above comoving coordinates $\{\widetilde{X}, \widetilde{Y}, \widetilde{Z}\}$, but for the rest of this overview let us suppress the tildes on these coordinates. Suppose a function $\psi(X, Y, Z)$ on the block is formally represented as a triple Chebyshev expansion

$$
\psi(X, Y, Z)=\sum_{n=0}^{\infty} \sum_{m=0}^{\infty} \sum_{p=0}^{\infty} \widetilde{\psi}_{n m p} T_{n}(\xi(X)) T_{m}(\eta(Y)) T_{p}(\chi(Z)),
$$

where $(\xi(X), \eta(Y), \chi(Z))$ maps our block to the standard cube $[-1,1]^{3}$. To get an approximation of $\psi(X, Y, Z)$, we consider the truncated series

$$
\mathcal{P}_{N_{X}, N_{Y}, N_{Z}} \psi(X, Y, Z)=\sum_{n=0}^{N_{X}} \sum_{m=0}^{N_{Y}} \sum_{p=0}^{N_{Z}} \widetilde{\psi}_{n m p} T_{n}(\xi(X)) T_{m}(\eta(Y)) T_{p}(\chi(Z)),
$$

so that $\psi(X, Y, Z)$ is represented (either exactly or approximately) by a three-index set $\left\{\widetilde{\psi}_{n m p}: 0 \leq n \leq N_{X}, 0 \leq m \leq N_{Y}, 0 \leq p \leq N_{Z}\right\}$ of modal coefficients. We represent this finite collection of modal coefficients as a column vector $\widetilde{\boldsymbol{\psi}}$, with components $\widetilde{\boldsymbol{\psi}}(\alpha)=(\widetilde{\boldsymbol{\psi}})_{\alpha}$ determined by the direct product representation 3

$$
\widetilde{\boldsymbol{\psi}}\left(n\left(N_{Y}+1\right)\left(N_{Z}+1\right)+m\left(N_{Z}+1\right)+p\right)=\widetilde{\psi}_{n m p} .
$$

A single matrix operating on the vector $\widetilde{\psi}$ (all modal coefficients representing the given function) may then equivalently be considered as having block-elements which are other matrices. We always view the modal set for a function on a cylindrical or rectangular subdomain as a direct product of three one-dimensional sets. However, in the case of the spherical shells $(J, H$, and $O$ ), we sometimes view the set of modal coefficients as the direct product of only two sets, the set corresponding to the radial modes and the set corresponding to the spherical harmonic modes (which involve both the polar and azimuthal angles).

In our notation, operators corresponding to a single dimension, that is "simple" matrices (whose elements are numbers, not matrices), are usually represented by a capital in an ordinary font, such as the identity operator/matrix $I_{X}$ or the matrix $D_{Z}$ which realizes differentiation by $Z$. Matrices which act on the full set of modal coefficients are represented by a calligraphic capital, for example $\mathcal{B}$. Thus, if $B_{Z[1]}$ represents integration in $Z$, then on a rectangular subdomain we might have $\mathcal{B}=I_{X} \otimes I_{Y} \otimes B_{Z[1]}$ as the matrix which, when applied to a vector $\widetilde{\boldsymbol{\psi}}$ holding the full set of modal coefficients, realizes integration in $Z$ with no action in $X$ or $Y$. That is, if $\psi(X, Y, Z)$ has modal coefficients $\widetilde{\boldsymbol{\psi}}$, then formally $\int \psi(X, Y, Z) d Z$ has modal coefficients $\mathcal{B} \widetilde{\boldsymbol{\psi}}$. The [1] on $B_{Z[1]}$ indicates that all entries in the first row (in fact,

${ }^{3}$ We could have instead taken

$$
\widetilde{\boldsymbol{\psi}}\left(m\left(N_{X}+1\right)\left(N_{Z}+1\right)+p\left(N_{X}+1\right)+n\right)=\widetilde{\psi}_{n m p},
$$

which might prove advantageous for representation of the $\Omega^{2}$ term in (8). Our choice (7) has been determined by technical decisions made during the initial construction of our code. In any case, based on some experimentation, we believe this choice makes little difference, at least for $\Omega \lesssim 0.5$ (well in the range of rotation rates we aspire to treat). 
the zeroth row by our conventions) of this matrix are zero, so that $(\mathcal{B} \widetilde{\boldsymbol{\psi}})(\alpha)=0$ whenever $p=0$ for the index $\alpha=n\left(N_{Y}+1\right)\left(N_{Z}+1\right)+m\left(N_{Z}+1\right)+p$ [cf. Eq. (7)]. This choice would fix the integration constant (a function of $X$ and $Y$ ) in $\int \psi(X, Y, Z) d Z$, but this empty row might also be subsequently filled with a "tau-condition," that is another vector chosen to fix a different constant.

\section{Integration preconditioning}

Let us briefly review the key ideas behind the technique of integration preconditioning, continuing to assume a rectangular block subdomain and also assuming the operator (41) for the HRWE. (See Refs. [1, 6] for nonlinear scenarios, [1] for more complicated 1D operators, and Refs. [1, 37] for more exotic basis functions). After enough invocations of the Leibniz rule, we may express the operator in (4) (again with tildes suppressed) as

$$
L=\partial_{Y}^{2}+\partial_{X}^{2}\left(1-\Omega^{2} Z^{2}\right)+\partial_{Z}^{2}\left(1-\Omega^{2} X^{2}\right)-\Omega^{2}\left(\partial_{X} X+\partial_{Z} Z-2 \partial_{X} \partial_{Z} X Z\right) .
$$

We view this equation as an operator identity, that is the partial derivatives see both terms like $Z^{2}$ and $X Z$ to the right, and also the function (not shown) on which $L$ will eventually act. The Chebyshev polynomials $T_{n}(\xi)$ obey the three-term recurrence $2 \xi T_{n}(\xi)=T_{n+1}(\xi)+$ $T_{n-1}(\xi)$. Here $\xi$ may be viewed as a suitable rescaling of either $X, Y$, or $Z$. Therefore, multiplication by the independent variable (here $\xi$ ) is represented in the corresponding space of modal coefficients by a banded (evidently a tridiagonal) matrix $A_{\xi}$. In fact, multiplication by a polynomial $p(\xi)$ is similarly represented by a banded matrix $p\left(A_{\xi}\right)$. Now, the matrix which represents $L$ is

$$
\begin{aligned}
\mathcal{L} & =I_{X} \otimes D_{Y}^{2} \otimes I_{Z}+D_{X}^{2} \otimes I_{Y} \otimes\left(I_{Z}-\Omega^{2} A_{Z}^{2}\right)+\left(I_{X}-\Omega^{2} A_{X}^{2}\right) \otimes I_{Y} \otimes D_{Z}^{2} \\
& -\Omega^{2}\left(D_{X} A_{X} \otimes I_{Y} \otimes I_{Z}+I_{X} \otimes I_{Y} \otimes D_{Z} A_{Z}-2 D_{X} A_{X} \otimes I_{Y} \otimes D_{Z} A_{Z}\right) .
\end{aligned}
$$

where each $D$ represents differentiation in the space of modal coefficients for one coordinate. This matrix is of the general form

$$
\mathcal{L}=\sum_{i=0}^{2} \sum_{j=0}^{2} \sum_{k=0}^{2} D_{X}^{i} \otimes D_{Y}^{j} \otimes D_{Z}^{k}\left(\sum_{r=0}^{2} \sum_{s=0}^{2} \sum_{t=0}^{2} p_{i j k, r s t} A_{X}^{r} \otimes A_{Y}^{s} \otimes A_{Z}^{t}\right),
$$

where the $p_{i j k, r s t}$ are constants (most zero in our case). In Eq. (10) the matrix within the parenthesis is banded and sparse; however, overall $\mathcal{L}$ is neither, since these desirable features are spoiled by the derivative matrices (see the second paragraph of this subsection).

The idea behind integration preconditioning is to "undo" all of the matrix differentiations which appear in (10) through repeated application of integration matrices [cf. point (iii) in the second paragraph of this subsection]. To illustrate, we consider the modal representation $\mathcal{L} \widetilde{\boldsymbol{\psi}}=\widetilde{\boldsymbol{g}}$ of (3) on the rectangular block, ignoring for the time being the issue of boundary conditions. Introducing $\mathcal{B} \equiv B_{X[2]}^{2} \otimes B_{Y[2]}^{2} \otimes B_{Z[2]}^{2}$, we then form $\mathcal{B} \mathcal{L} \widetilde{\boldsymbol{\psi}}=\mathcal{B} \widetilde{\boldsymbol{g}}$. The coefficient matrix of the new "preconditioned" system is then

$$
\begin{aligned}
& \mathcal{B L}=B_{X[2]}^{2} \otimes I_{Y[2]} \otimes B_{Z[2]}^{2} \\
& +I_{X[2]} \otimes B_{Y[2]}^{2} \otimes\left(B_{Z[2]}^{2}-\Omega^{2} B_{Z[2]}^{2} A_{Z}^{2}\right)+\left(B_{X[2]}^{2}-\Omega^{2} B_{X[2]}^{2} A_{X}^{2}\right) \otimes B_{Y[2]}^{2} \otimes I_{Z[2]} \\
& -\Omega^{2}\left(B_{X[2]} A_{X} \otimes B_{Y[2]}^{2} \otimes B_{Z[2]}^{2}+B_{X[2]}^{2} \otimes B_{Y[2]}^{2} \otimes B_{Z[2]} A_{Z}-2 B_{X[2]} A_{X} \otimes B_{Y[2]}^{2} \otimes B_{Z[2]} A_{Z}\right) .
\end{aligned}
$$




\begin{tabular}{|l|l|l|}
\hline \multicolumn{1}{|c|}{ Face } & \multicolumn{1}{|c|}{ Rows } & \multicolumn{1}{c|}{ Index restrictions } \\
\hline$Z=Z_{\min }$ & $n\left(N_{Y}+1\right)\left(N_{Z}+1\right)+m\left(N_{Z}+1\right)+0$ & $0 \leq n \leq N_{X}, 0 \leq m \leq N_{Y}$ \\
$Z=Z_{\max }$ & $n\left(N_{Y}+1\right)\left(N_{Z}+1\right)+m\left(N_{Z}+1\right)+1$ & $0 \leq n \leq N_{X}, 0 \leq m \leq N_{Y}$ \\
\hline$Y=Y_{\min }$ & $n\left(N_{Y}+1\right)\left(N_{Z}+1\right)+p$ & $0 \leq n \leq N_{X}, 2 \leq p \leq N_{Z}$ \\
$Y=Y_{\max }$ & $n\left(N_{Y}+1\right)\left(N_{Z}+1\right)+\left(N_{Z}+1\right)+p$ & $0 \leq n \leq N_{X}, 2 \leq p \leq N_{Z}$ \\
\hline$X=X_{\min }$ & $m\left(N_{Z}+1\right)+p$ & $2 \leq m \leq N_{Y}, 2 \leq p \leq N_{Z}$ \\
$X=X_{\max }$ & $\left(N_{Y}+1\right)\left(N_{Z}+1\right)+m\left(N_{Z}+1\right)+p$ & $2 \leq m \leq N_{Y}, 2 \leq p \leq N_{Z}$ \\
\hline
\end{tabular}

TABLE II. FILLING OF EMPTY ROWS FOR BLOCKS.

Because it is built only with $B \mathrm{~s}$ and $A$ s, this matrix is sparse and banded, albeit with large bandwidth due to the direct product structure.

The matrix $\mathcal{B} \mathcal{L}$ has many empty rows, signaling missing information. The spectral-tau procedure is to put the "tau conditions," here the boundary conditions, in these empty rows, and the corresponding inhomogeneous values in $\mathcal{B} \widetilde{\boldsymbol{g}}$. When this procedure is carried out correctly, with due regard to possible repetition in the specification of boundary data, the empty rows provide precisely the space needed for the boundary data of a well-posed problem. To enforce boundary conditions for the example at hand, we proceed as follows. Define, for example, $h^{+}(X, Y)=\psi\left(X, Y, Z_{\max }\right)$ and $h^{-}(X, Y)=\psi\left(X, Y, Z_{\min }\right)$. Then Dirichlet boundary conditions along the $X Y$-faces of a block are expressible as

$$
\sum_{p=0}^{N_{Z}} \widetilde{\psi}_{n m p} \delta_{p}^{ \pm}=\tilde{h}_{n m}^{ \pm}
$$

where a double Chebyshev projection appears on the right-hand side. Moreover, $\delta^{+}$(all 1's) and $\delta^{-}$(alternating +1 and -1 ) are the $\left(N_{Z}+1\right)$ dimensional "Dirichlet vectors." Similar equations correspond to $Y Z$ and $X Y$ faces, and in all $2\left(N_{X}+1\right)\left(N_{Y}+1\right)+2\left(N_{Y}+1\right)\left(N_{Z}+\right.$ $1)+2\left(N_{X}+1\right)\left(N_{Z}+1\right)$ such equations are possible. However, there are only

$$
2\left(N_{X}+1\right)\left(N_{Y}+1\right)+2\left(N_{Y}+1\right)\left(N_{Z}+1\right)+2\left(N_{X}+1\right)\left(N_{Z}+1\right)-4\left(N_{X}+N_{Y}+N_{Z}+1\right)
$$

available empty rows in (11). However, there are precisely $4\left(N_{X}+N_{Y}+N_{Z}+1\right)$ linear dependencies amongst the set of all possible boundary equations, owing to the fact that faces share common edge values. Table II gives our prescription for filling empty rows.

As a result of the integration preconditioning, we have reformulated the set of equations in terms of matrices with a drastic reduction in nonzero elements. In the context of ODEs, that is in the 1D origins of this method, Ref. [1] has thoroughly studied the condition number of the resulting preconditioned matrix with respect to norms that arise from diagonal equilibration. While in ODE settings integration preconditioning often improves the conditioning of the original system, in the PDE context at hand $B_{X[2]}^{2} \otimes B_{Y[2]}^{2} \otimes B_{Z[2]}^{2}$ is not an optimal preconditioner in that it does not approximate (in any measure that we are aware of) the inverse of the original coefficient matrix. Nevertheless, one should expect that the "preconditioned" coefficient matrix has a more clustered spectrum, since the Bs are compact operators (even as infinite dimensional matrices). A clustered spectrum often yields favorable convergence properties in the context of Krylov iterative methods. Regardless, sparsification is a desirable property, since it clearly affords a fast matrix-vector multiply in Krylov methods. Therefore, for multidimensional problems we are more comfortable 
focusing on the sparsifying aspect of the technique, with the understanding that further preconditioning (described below) on top of the "integration preconditioning" will be required to enhance convergence of the underlying linear solver (in our case GMRES).

\section{SPARSE SPECTRAL APPROXIMATION OF THE 3D HRWE}

Starting with one of the forms appearing in (41) and after further transformations, this section describes the spectral-tau representation of $L$ on each of the basic subdomains, except for the block case which we have already described in Sec. IC. These descriptions allow for implementation of the lefthand side of (3) as a "matrix-vector multiply," an implementation required by the iterative solver GMRES [35].

\section{A. Outer spherical shell}

In the polar system associated with the comoving system (1) the operator (4) becomes

$$
r^{2} L=r^{2} \Delta-\Omega^{2} J_{O}, \quad r^{2} \Delta=\partial_{r}^{2} r^{2}-2 \partial_{r} r+\Delta_{S^{2}}, \quad J_{O}=r^{2} \partial_{\varphi}^{2},
$$

where $\Delta_{S^{2}}$ is the unit-sphere Laplacian and $O$ stands for the outer spherical shell. These equations should be viewed as operator identities acting on scalar functions. The solution $\psi$ to $L \psi=g$ is formally represented as the triple series

$$
\begin{aligned}
\psi(r, \theta, \varphi) & =\sum_{n=0}^{\infty} \sum_{\ell=0}^{\infty} \widetilde{\psi}_{\ell 0 n} \bar{P}_{\ell 0}(\cos \theta) T_{n}(\xi(r)) \\
& +\sum_{n=0}^{\infty} \sum_{\ell=1}^{\infty} \sum_{m=1}^{\ell} \bar{P}_{\ell m}(\cos \theta)\left[\widetilde{\psi}_{\ell, 2 m-1, n} \cos (m \varphi)+\widetilde{\psi}_{\ell, 2 m, n} \sin (m \varphi)\right] T_{n}(\xi(r)),
\end{aligned}
$$

where the $\bar{P}_{\ell m}(\cos \theta)$ are normalized associated Legendre functions [38] and $\xi(r)$ maps the radial domain to the standard interval $[-1,1]$. The corresponding numerical approximation is the following finite expansion:

$$
\begin{aligned}
\mathcal{P}_{N_{r}, N_{\theta}} \psi(r, \theta, \varphi) & =\sum_{n=0}^{N_{r}} \sum_{\ell=0}^{N_{\theta}} \widetilde{\psi}_{\ell 0 n} \bar{P}_{\ell 0}(\cos \theta) T_{n}(\xi(r)) \\
& +\sum_{n=0}^{N_{r}} \sum_{\ell=1}^{N_{\theta}} \sum_{m=1}^{N_{\theta}} \bar{P}_{\ell m}(\cos \theta)\left[\widetilde{\psi}_{\ell, 2 m-1, n} \cos (m \varphi)+\widetilde{\psi}_{\ell, 2 m, n} \sin (m \varphi)\right] T_{n}(\xi(r)) .
\end{aligned}
$$

We represent the triply-indexed modal coefficients $\widetilde{\psi}_{\ell q n}$ as a vector $\widetilde{\psi}(\alpha)$ of length $\left(N_{\theta}+\right.$ 1) $\left(2 N_{\theta}+1\right)\left(N_{r}+1\right)$, with the two notations connected by

$$
\widetilde{\boldsymbol{\psi}}\left(\ell\left(2 N_{\theta}+1\right)\left(N_{r}+1\right)+q\left(N_{r}+1\right)+n\right)=\widetilde{\psi}_{\ell q n},
$$

For $\ell<N_{\theta}$ the second sum over $m$ in (15) includes too many terms. Indeed, $m$ should run from 1 to $\ell$ only (with the $m=0$ terms appearing in the first sum); therefore, whenever 
$q>2 \ell$, we must set $\widetilde{\psi}_{\ell q n}=0$ by hand. We have enlarged the space of modal coefficients for later convenience when using spherical harmonic transformations. With this remark in mind, for our representation (16) the index $\alpha$ of the vector $\widetilde{\psi}(\alpha)$ starts at 0 and takes on all values corresponding to the ranges $0 \leq \ell \leq N_{\theta}, 0 \leq q \leq 2 N_{\theta}$, and $0 \leq n \leq N_{r}$. We denote by $\mathbb{P}$ the projection matrix whose range is the set of vectors associated with proper spherical harmonic expansions,

$$
(\mathbb{P} \widetilde{\boldsymbol{\psi}})\left(\ell\left(2 N_{\theta}+1\right)\left(N_{r}+1\right)+q\left(N_{r}+1\right)+n\right)=0, \text { for } q>2 \ell .
$$

Let us first consider a sparse approximation of the Laplacian term $r^{2} \Delta$, which from (13) has the spectral representation

$$
\mathcal{A}_{r}^{2} \boldsymbol{\Delta}=\mathbb{P}\left[I_{\theta} \otimes I_{\varphi} \otimes\left(D_{r}^{2} A_{r}^{2}-2 D_{r} A_{r}\right)-\mathscr{L}^{2} \otimes I_{r}\right],
$$

where $\mathcal{A}_{r}^{2}=I_{\theta} \otimes I_{\varphi} \otimes A_{r}^{2}$, and $A_{r}$ is the matrix equivalent to multiplying $r$-dependent functions by a factor of $r$. In the first term within the square brackets $I_{\theta} \otimes I_{\varphi} \otimes$ means that there are no operations mixing modes $\widetilde{\psi}_{\ell q n}$ with different values of $\ell$, or of $q$ (i.e. the dual indices to $\theta$ and $\phi)$. The operator $\left(D_{r}^{2} A_{r}^{2}-2 D_{r} A_{r}\right)$ is the matrix equivalent of the partial differentiation $\partial_{r}^{2} r^{2}-2 \partial_{r} r$ in (13). The matrix $\mathscr{L}^{2}$, representing $-\Delta_{S^{2}}$ in (13), is comprised of $\left(N_{\theta}+1\right)$ constant blocks $\ell(\ell+1) I_{\left(2 N_{\theta}+1\right) \times\left(2 N_{\theta}+1\right)}$ in each subspace labeled by $\ell$.

To get a sparse form of the Laplacian, we define $\mathcal{B}=I_{\theta} \otimes I_{\varphi} \otimes B_{r}^{2}$ and write the expression

$$
\left(\mathcal{B A}_{r}^{2} \boldsymbol{\Delta}\right)^{\text {modified }}=\mathbb{P}\left[I_{\theta} \otimes I_{\varphi} \otimes\left(I_{r[2]} A_{r}^{2}-2 B_{r[2]} A_{r}\right)+\mathscr{L}^{2} \otimes B_{r[2]}^{2}\right]+\left(I_{\theta} \otimes I_{\varphi} \otimes I_{r}-\mathbb{P}\right) .
$$

Here the "modified" notation indicates that, by the addition of the last term above, 1's have been placed on the diagonal in rows set to zero by the projection operation, so that the result is a nonsingular matrix. Therefore, to ensure that a solution $\widetilde{\boldsymbol{\psi}}$ to the corresponding linear system obeys

$$
\widetilde{\psi}\left(\ell\left(2 N_{\theta}+1\right)\left(N_{r}+1\right)+q\left(N_{r}+1\right)+n\right)=0, \quad \text { for } q>2 \ell .
$$

We demand that the source obeys $\widetilde{\boldsymbol{g}}=\mathbb{P} \widetilde{\boldsymbol{g}}$. Finally, from (13) the sparse preconditioned form of the operator $J_{O}$ is

$$
\mathcal{B} \mathcal{J}_{O}=-\mathbb{P}\left[I_{\theta} \otimes \mathscr{M}^{2} \otimes B_{r[2]}^{2} A_{r}^{2}\right]
$$

where $\mathscr{M}^{2}=\operatorname{diag}\left(0,1,1,4,4, \cdots, N_{\theta}^{2}, N_{\theta}^{2}\right)$ is the $\left(2 N_{\theta}+1\right)$-by- $\left(2 N_{\theta}+1\right)$ matrix representing $-\partial_{\varphi}^{2}$. Therefore, $\left(\mathcal{B A}_{r}^{2} \boldsymbol{\Delta}\right)^{\text {modified }}-\Omega^{2} \mathcal{B} \mathcal{J}_{O}$ is our sparse form of the overall coefficient matrix, prior to inclusion of boundary conditions.

We now consider specification of outer radiation conditions, for which we summarize results given in [6]. Specification of Dirichlet conditions on the inner boundary $\partial O^{-}$of the outer shell $O$ is essentially the same as specification on the boundaries $\partial H^{ \pm}$of the inner shell $H$, and we describe that specification in detail below. The specification at $\partial O^{+}$, however, involves radiation conditions. To define these, we set $R=r_{\max }$, the radial coordinate value of $\partial \mathrm{O}^{+}$, and introduce

$$
V_{\ell+1 / 2}(\xi)=\sqrt{\frac{\pi \xi}{2}} \exp \left[-\mathrm{i}\left(\xi-\frac{1}{2} \pi \kappa-\frac{1}{4} \pi\right)\right] H_{\ell+1 / 2}^{(+)}(\xi)
$$


which satisfies $V_{\ell+1 / 2}(\xi) \sim 1$ as $\xi \rightarrow \infty$. Here $H_{\ell+1 / 2}^{(+)}(\xi)$ is the cylindrical Hankel function of the first kind, of half-integer order $\ell+1 / 2$. For our radiative boundary condition we will need the "frequency-domain kernel,"

$$
v_{\ell+1 / 2}(\xi) \equiv \xi \frac{V_{\ell+1 / 2}^{\prime}(\xi)}{V_{\ell+1 / 2}(\xi)},
$$

which is computable as a continued fraction via Steed's algorithm [39]. Radiation conditions involve

$$
p \equiv m \Omega R+\operatorname{Im}\left(v_{\ell+1 / 2}(m \Omega R)\right), \quad q \equiv 1-\operatorname{Re}\left(v_{\ell+1 / 2}(m \Omega R)\right),
$$

with $p=0$ and $q=\ell+1$ for $m=0$ modes (see Ref. [6] for details). The $p$ and $q$ here (in particular the $q$ ) are not related to the indices on $\widetilde{\psi}_{\ell q n}$. Both uses of $p$ and $q$ will not appear in the same formula. As tau-conditions, our radiation conditions are then expressible as

$$
\begin{gathered}
\sum_{n=0}^{N_{r}}\left(R \widetilde{\psi}_{\ell, 2 m, n} \nu_{n}^{+}+p \widetilde{\psi}_{\ell, 2 m-1, n} \delta_{n}^{+}+q \widetilde{\psi}_{\ell, 2 m, n} \delta_{n}^{+}\right)=0 \\
\sum_{n=0}^{N_{r}}\left(R \widetilde{\psi}_{\ell, 2 m-1, n} \nu_{n}^{+}-p \widetilde{\psi}_{\ell, 2 m, n} \delta_{n}^{+}+q \widetilde{\psi}_{\ell, 2 m-1, n} \delta_{n}^{+}\right)=0 .
\end{gathered}
$$

Here $\delta^{+}$(all 1's) and $\delta^{-}$(alternating +1 and -1$)$ are the $\left(N_{r}+1\right)$ dimensional "Dirichlet vectors" used to impose Dirichlet conditions at the endpoints of a coordinate range. Similarly, $\nu^{+}$and $\nu^{-}$are the $\left(N_{r}+1\right)$ dimensional "Neumann vectors" used to impose derivative conditions at the endpoints. Details are given in [1, 6].

Along the block-diagonal of the coefficient matrix $\left(\mathcal{B A}_{r}^{2} \boldsymbol{\Delta}\right)^{\text {modified }}-\Omega^{2} \mathcal{B} \mathcal{J}_{O}$, there are $\left(N_{r}+1\right)$-by- $\left(N_{r}+1\right)$ blocks, one for each $(\ell, q)$ pair. When $q$ exceeds $2 \ell$, each such block is the identity matrix; however, the block corresponding to a physical mode $0 \leq q \leq 2 \ell$ has the form

$$
\left[\begin{array}{c}
\mathbf{0} \\
\mathbf{0} \\
\hline \mathrm{B}^{\ell q}
\end{array}\right]
$$

Here 0 represents a row of zeros, and $\mathrm{B}^{\ell q}$ is a nonzero $\left(N_{r}-1\right)$-by- $\left(N_{r}+1\right)$ submatrix. The zeros in the first two rows are filled in with the Dirichlet boundary conditions on $\partial O^{-}$, using $\delta^{-}$, and the radiation boundary conditions on $\partial O^{+}$, using (25). Since these radiation conditions mix one cosine $(q=2 m-1)$ and the other sine $(q=2 m)$ mode, the tau conditions lead to a coupling between among the blocks. The resulting $2\left(N_{r}+1\right)$-by-2 $\left(N_{r}+1\right)$ block neighborhood, with Dirichlet and radiation boundary conditions, takes one of the following forms (either representation is possible due to the homogeneity of the boundary conditions):

$$
\left[\begin{array}{c|c}
\delta^{-} & \mathbf{0} \\
p \delta^{+} & R \nu^{+}+q \delta^{+} \\
\mathrm{B}^{\ell, 2 m-1} & \mathbf{0} \\
\hline \mathbf{0} & \delta^{-} \\
R \nu^{+}+q \delta^{+} & -p \delta^{+} \\
\mathbf{0} & \mathrm{B}^{\ell, 2 m}
\end{array}\right] \quad \text { or }\left[\begin{array}{c|c}
\delta^{-} & \mathbf{0} \\
R \nu^{+}+q \delta^{+} & -p \delta^{-} \\
\mathrm{B}^{\ell, 2 m-1} & \mathbf{0} \\
\hline \mathbf{0} & \delta^{-} \\
p \delta^{+} & R \nu^{+}+q \delta^{+} \\
\mathbf{0} & \mathrm{B}^{\ell, 2 m}
\end{array}\right]
$$


where 0 represents either a row (when opposite a $\left.\delta^{-}\right)$or a $\left(N_{r}-1\right)$-by- $\left(N_{r}+1\right)$ submatrix of zeros (when opposite a B). Boundary conditions for $m=0$ (zero modes) correspond to blocks

$$
\left[\begin{array}{c}
\delta^{-} \\
R \nu^{+}+q \delta^{+} \\
\mathrm{B}^{\ell 0}
\end{array}\right]
$$

Evidently, in this case only a single azimuthal block need be considered.

\section{B. Inner spherical shells}

We stress that the polar coordinates $(r, \theta, \phi)$ appearing in this subsection are not the polar coordinates $(r, \theta, \varphi)$ used in the last, although only the notation for the azimuthal angle ( $\phi$ vs. $\varphi$ ) reflects the difference. We start with (4), assume that one of the "holes" is at $\widetilde{Z}=z_{H}$, and define new comoving coordinates

$$
z=\widetilde{Z}-z_{H}, \quad x=\widetilde{X}, \quad y=\widetilde{Y} .
$$

The helically reduced wave operator in the new coordinates is

$$
L=\frac{\partial^{2}}{\partial x^{2}}+\frac{\partial^{2}}{\partial y^{2}}+\frac{\partial^{2}}{\partial z^{2}}-\Omega^{2}\left[\left(z_{H}+z\right) \frac{\partial}{\partial x}-x \frac{\partial}{\partial z}\right]^{2} .
$$

Spherical polar coordinates $\{r, \theta, \phi\}$ in this subsection correspond to the system $\{x, y, z\}$.

As already mentioned, the system $\{r, \theta, \phi\}$ is not the system $\{r, \theta, \varphi\}$ corresponding the outer shell. Nevertheless, for an inner shell our treatment of the Laplacian part of the operator is the same as the treatment given in the last subsection. In particular, we adopt the same conventions for the indexing of the spectral representation, and therefore again arrive at the expression (19). Notationally, the only difference is that we replace all instances of $\varphi$ with $\phi$. Therefore, having already considered $\left(r^{2} \times\right)$ the Laplacian part of the HRWE, we turn to $\left(r^{2} \times\right)$ the term in (30) paired with $-\Omega^{2}$,

$$
J_{H} \equiv r^{2}\left[\left(z_{H}+z\right) \partial / \partial x-x \partial / \partial z\right]^{2} .
$$

To facilitate the expression of the derivatives in (31) in terms of operations on $\{r, \theta, \phi\}$, we introduce

$$
\begin{aligned}
& Q=\sin \theta \cos \phi \\
& P=\cos \theta \cos \phi \partial / \partial \theta-\csc \theta \sin \phi \partial / \partial \phi \\
& N=\cos \phi \partial / \partial \theta-\cos \theta \csc \theta \sin \phi \partial / \partial \phi,
\end{aligned}
$$

and note that

$$
\partial / \partial x=Q \partial / \partial r+r^{-1} P, \quad z \partial / \partial x-x \partial / \partial z=N .
$$

With the identities (which should be read as operators acting on a scalar function)

$$
r^{2} \partial_{r}^{2}=\partial_{r}^{2} r^{2}-4 \partial_{r} r+2, \quad r \partial_{r}=\partial_{r} r-1, \quad r^{2} \partial_{r}=\partial_{r} r^{2}-2 r
$$


we then find that $J_{H}$ of (31) can be written as

$$
\begin{aligned}
J_{H} & =z_{H}^{2} Q^{2} \partial_{r}^{2} r^{2}+z_{H}^{2}\left(P Q+Q P-4 Q^{2}\right) \partial_{r} r+z_{H}(N Q+Q N) \partial_{r} r^{2} \\
& +z_{H}(N P+P N-2 N Q-2 Q N) r+N^{2} r^{2} \\
& +z_{H}^{2}\left(P^{2}+2 Q^{2}-P Q-2 Q P\right) .
\end{aligned}
$$

We use $\mathcal{J}_{H}$ to denote the spectral form of the differential operator $J_{H}$. The corresponding sparse form $\mathcal{B J}_{H} \equiv\left(I_{\theta} \otimes I_{\phi} \otimes B_{r[2]}^{2}\right) \mathcal{J}_{H}$ is then

$$
\begin{aligned}
\mathcal{B J}_{H} & =z_{H}^{2} \mathrm{Q}^{2} \otimes I_{[2] r} A_{r}^{2}+z_{H}^{2}\left(\mathrm{PQ}+\mathrm{QP}-4 \mathrm{Q}^{2}\right) \otimes B_{[2] r} A_{r} \\
& +z_{H}(\mathrm{NQ}+\mathrm{QN}) \otimes B_{[2] r} A_{r}^{2}+z_{H}(\mathrm{NP}+\mathrm{PN}-2(\mathrm{NQ}+\mathrm{QN})) \otimes B_{[2] r}^{2} A_{r} \\
& +\mathrm{N}^{2} \otimes B_{[2] r}^{2} A_{r}^{2}+z_{H}^{2}(\mathrm{P}-2 \mathrm{Q})(\mathrm{P}-\mathrm{Q}) \otimes B_{[2] r}^{2} .
\end{aligned}
$$

Here the san serif N, P, and $Q$ are matrices acting on the spectral space of spherical harmonic expansion coefficients. Whence we need explicit realizations of the following matrices: $Q^{2}$, $\mathrm{PQ}+\mathrm{QP}, \mathrm{PN}+\mathrm{NP}, \mathrm{PQ}+\mathrm{QP}$, and $(\mathrm{P}-\mathrm{Q})^{2}$. We compute these matrices as truncations of the corresponding exact infinite dimensional matrices described below (with products computed before truncation). The truncated matrix components $\mathbf{N}(\alpha, \beta)$ of $\mathbf{N}$ obey the following condition:

$$
\mathrm{N}\left(\ell\left(2 N_{\theta}+1\right)+q, k\left(2 N_{\theta}+1\right)+p\right)=0, \quad \text { for } q>2 \ell \text { or } p>2 k,
$$

and similarly for the components $\mathrm{P}(\alpha, \beta)$ and $\mathrm{Q}(\alpha, \beta)$. (Here we have switched to parenthesis notation [36] for the components $\mathrm{N}(i, j)=\mathrm{N}_{i j}$ of a matrix.) This condition properly treats the extraneous components we have included in our expansion vector $\widetilde{\boldsymbol{\psi}}$.

Of the three angular differential operators $P, Q, N$, we only consider $N$ in detail here, as its action on spherical harmonics is the simplest to describe. Partial formulas are given for $P$ and $Q$ at the end of this subsection. Using standard formulas from the theory of angular momentum (see the appendix of [40]), we have

$$
N Y_{\ell m}=\frac{1}{2} \sqrt{(\ell-m)(\ell+m+1)} Y_{\ell, m+1}-\frac{1}{2} \sqrt{(\ell+m)(\ell-m+1)} Y_{\ell, m-1} .
$$

Before completing our construction of N, P, and Q, we first collect some formulas which relate the standard complex representation of spherical harmonics $Y_{\ell m}(\theta, \phi)$ to the realvalued representation. The normalized Legendre functions are [38]

$$
\bar{P}_{\ell m}(u)=(-1)^{m} \sqrt{\frac{2 \ell+1}{2} \frac{(\ell-m) !}{(\ell+m) !}} P_{\ell}^{m}(u),
$$

with $P_{\ell}^{m}(u)$ the standard associated Legendre function (as given, for example, by Thorne [41]). We then have

$$
Y_{\ell m}=\sqrt{\frac{1}{2 \pi}}(-1)^{m} \bar{P}_{\ell m} e^{\mathrm{i} m \phi}, \quad Y_{\ell,-m}=\sqrt{\frac{1}{2 \pi}} \bar{P}_{\ell m} e^{-\mathrm{i} m \phi}, \quad m \geq 0 .
$$

For fixed $\ell$, the expansion in azimuthal index takes the form

$$
c_{\ell 0} Y_{\ell 0}+\sum_{m=1}^{\ell}\left(c_{\ell m} Y_{\ell m}+c_{\ell,-m} Y_{\ell,-m}\right)=a_{\ell 0} \bar{P}_{\ell 0}+\sum_{m=1}^{\ell} \bar{P}_{\ell m}\left[a_{\ell m} \cos m \phi+b_{\ell m} \sin m \phi\right],
$$


where the real expansion coefficients are $\sqrt{2 \pi} a_{\ell 0}=c_{\ell 0}$ and, for $m \geq 1$,

$$
\sqrt{2 \pi} a_{\ell m}=c_{\ell m}(-1)^{m}+c_{\ell,-m}, \quad \sqrt{2 \pi} b_{\ell m}=\mathrm{i}\left[c_{\ell m}(-1)^{m}-c_{\ell,-m}\right] .
$$

We define another set of complex expansion coefficients

$$
f_{\ell m}=\frac{1}{2} \sqrt{(\ell+m)(\ell-m+1)} c_{\ell, m-1}-\frac{1}{2} \sqrt{(\ell-m)(\ell+m+1)} c_{\ell, m+1},
$$

so that, from (40), the action of $N$ has the effect

$$
\Psi=\sum_{\ell=0}^{\infty} \sum_{m=-\ell}^{\ell} c_{\ell m} Y_{\ell m}, \quad N \Psi=\sum_{\ell=0}^{\infty} \sum_{m=-\ell}^{\ell} f_{\ell m} Y_{\ell m} .
$$

We can then represent $N$ by the matrix $\mathrm{N}$ that converts the vector of coefficients $c_{\ell m}$ to the vector $f_{\ell m}$ by $\boldsymbol{f}=\mathrm{N} \boldsymbol{c}$. We also define real coefficients $d_{\ell m}, e_{\ell m}$ which are related to $f_{\ell m}$ in the same way that $a_{\ell m}, b_{\ell m}$ are related to $c_{\ell m}$, and then view the action of $\mathrm{N}$ as a mapping from the real coefficients $a_{\ell m}, b_{\ell m}$ to the real coefficients $d_{\ell m}, e_{\ell m}$.

Turning to the representation for $Q$, we likewise use results tabulated in the appendix of [40] to find

$$
\begin{aligned}
Q Y_{\ell m} & =\frac{1}{2} \sqrt{\frac{(\ell-m+1)(\ell-m+2)}{(2 \ell+1)(2 \ell+3)}} Y_{\ell+1, m-1} \\
& -\frac{1}{2} \sqrt{\frac{(\ell+m+1)(\ell+m+2)}{(2 \ell+1)(2 \ell+3)}} Y_{\ell+1, m+1} \\
& -\frac{1}{2} \sqrt{\frac{(\ell+m)(\ell+m-1)}{(2 \ell+1)(2 \ell-1)}} Y_{\ell-1, m-1} \\
& +\frac{1}{2} \sqrt{\frac{(\ell-m)(\ell-m-1)}{(2 \ell+1)(2 \ell-1)}} Y_{\ell-1, m+1},
\end{aligned}
$$

so that $\boldsymbol{f}=\mathrm{Q} \boldsymbol{c}$ is determined by

$$
\begin{aligned}
f_{\ell m} & =\frac{1}{2} \sqrt{\frac{(\ell-m-1)(\ell-m)}{(2 \ell-1)(2 \ell+1)}} c_{\ell-1, m+1} \\
& -\frac{1}{2} \sqrt{\frac{(\ell+m-1)(\ell+m)}{(2 \ell-1)(2 \ell+1)}} c_{\ell-1, m-1} \\
& -\frac{1}{2} \sqrt{\frac{(\ell+m+2)(\ell+m+1)}{(2 \ell+1)(2 \ell+3)}} c_{\ell+1, m+1} \\
& +\frac{1}{2} \sqrt{\frac{(\ell-m+2)(\ell-m+1)}{(2 \ell+1)(2 \ell+3)}} c_{\ell+1, m-1} .
\end{aligned}
$$




\begin{tabular}{|c|c|c|}
\hline Boundary & Rows & Index restrictions \\
\hline$r=r_{\min }$ & $\ell\left(2 N_{\theta}+1\right)\left(N_{r}+1\right)+q\left(N_{r}+1\right)+0$ & $0 \leq \ell \leq N_{\theta}, 0 \leq q \leq 2 \ell$ \\
$r=r_{\max }$ & $\ell\left(2 N_{\theta}+1\right)\left(N_{r}+1\right)+q\left(N_{r}+1\right)+1$ & $0 \leq \ell \leq N_{\theta}, 0 \leq q \leq 2 \ell$ \\
\hline
\end{tabular}

TABLE III. FILLING OF EMPTY ROWS FOR SHELLS.

Again, we may express the action of $\mathrm{Q}$ as a mapping from $a_{\ell m}, b_{\ell m}$ to $d_{\ell m}, e_{\ell m}$. Finally, we use the identity

$$
\begin{aligned}
P Y_{\ell m}= & -\frac{1}{2} \ell \sqrt{\frac{(\ell-m+1)(\ell-m+2)}{(2 \ell+1)(2 \ell+3)}} Y_{\ell+1, m-1} \\
& +\frac{1}{2} \ell \sqrt{\frac{(\ell+m+1)(\ell+m+2)}{(2 \ell+1)(2 \ell+3)}} Y_{\ell+1, m+1} \\
& -\frac{1}{2}(\ell+1) \sqrt{\frac{(\ell+m)(\ell+m-1)}{(2 \ell+1)(2 \ell-1)}} Y_{\ell-1, m-1} \\
& +\frac{1}{2}(\ell+1) \sqrt{\frac{(\ell-m)(\ell-m-1)}{(2 \ell+1)(2 \ell-1)}} Y_{\ell-1, m+1} .
\end{aligned}
$$

to similarly define the action of $\mathrm{P}$ as a mapping from $a_{\ell m}, b_{\ell m}$ to $d_{\ell m}, e_{\ell m}$.

To enforce the inner and outer boundary conditions in (3), we fill empty rows in $\left(\mathcal{B A}_{r}^{2} \Delta\right)^{\text {modified }}-\Omega^{2} \mathcal{B J}_{H}$ with the tau-conditions. Let $h^{+}(\theta, \phi)=\psi\left(r_{\max }, \theta, \phi\right)$ and $h^{-}(\theta, \phi)=$ $\psi\left(r_{\min }, \theta, \phi\right)$. Then Dirichlet boundary conditions on the inner and outer boundaries of the shell are expressible as

$$
\sum_{n=0}^{N_{r}} \widetilde{\psi}_{\ell q n} \delta_{n}^{ \pm}=\tilde{h}_{\ell q}^{ \pm}
$$

where spherical-harmonic projections appear on the righthand side. Table III shows how empty rows are filled to enforce these boundary conditions.

\section{Cylindrical shells}

Throughout this section we suppress the tildes on $\tilde{X}, \widetilde{Y}, \widetilde{Z}$. Let $\rho=\left(X^{2}+Y^{2}\right)^{1 / 2}$, and multiply Eq. (4) by $\rho^{2}$ to get the operator identity

$$
\rho^{2} L=\rho^{2}\left[\partial_{Y}^{2}+\partial_{X}^{2}+\partial_{Z}^{2}-\Omega^{2} \partial_{X}^{2} Z^{2}-\Omega^{2} \partial_{Z}^{2} X^{2}-\Omega^{2}\left(\partial_{X} X+\partial_{Z} Z-2 \partial_{X} X \cdot \partial_{Z} Z\right)\right] .
$$

Since $X=\rho \cos \phi$ and $Y=\rho \sin \phi$,

$$
\rho^{2}\left(\partial_{X}^{2}+\partial_{Y}^{2}+\partial_{Z}^{2}\right)=\partial_{\rho}^{2} \rho^{2}-3 \partial_{\rho} \rho+1+\partial_{\phi}^{2}+\rho^{2} \partial_{Z}^{2},
$$

again with the view that this is an operator identity. Eq. (52) has the spectral representation

$$
\mathcal{A}_{\rho}^{2} \boldsymbol{\Delta}=I_{\phi} \otimes\left(I_{\rho}+D_{\rho}^{2} A_{\rho}^{2}-3 D_{\rho} A_{\rho}\right) \otimes I_{Z}+D_{\phi}^{2} \otimes I_{\rho} \otimes I_{Z}+I_{\phi} \otimes A_{\rho}^{2} \otimes D_{Z}^{2} .
$$

Here $\mathcal{A}_{\rho}^{2}$ represents the matrix $I_{\phi} \otimes A_{\rho}^{2} \otimes I_{Z}$. To achieve this representation we start by introducing a mapping of $\left[Z_{\min }, Z_{\max }\right]$ to $[-1,1]$ with the function $\chi(Z)$, so that $Z$ dependence 
can be expressed with the Chebyshev polynomials $T_{p}(\chi(Z))$. Similarly $\xi(\rho)$ maps $\left[\rho_{\min }, \rho_{\max }\right]$ to $[-1,1]$. The solution is then formally expressed as

$$
\begin{aligned}
\psi(\rho, \phi, Z) & =\sum_{n=0}^{\infty} \sum_{p=0}^{\infty} \widetilde{\psi}_{0 n p} T_{n}(\xi(\rho)) T_{p}(\chi(Z)) \\
& +\sum_{k=1}^{\infty} \sum_{n=0}^{\infty} \sum_{p=0}^{\infty}\left[\widetilde{\psi}_{2 k-1, n p} \cos (k \theta)+\widetilde{\psi}_{2 k, n p} \sin (k \theta)\right] T_{n}(\xi(\rho)) T_{p}(\chi(Z))
\end{aligned}
$$

with corresponding numerical truncation (taking $N_{\phi}$ even for simplicity)

$$
\begin{aligned}
\mathcal{P}_{N_{\rho}, N_{\phi}, N_{z}} \psi(\rho, \phi, Z) & =\sum_{n=0}^{N_{\rho}} \sum_{p=0}^{N_{z}} \widetilde{\psi}_{0 n p} T_{n}(\xi(\rho)) T_{p}(\chi(Z)) \\
& +\sum_{k=1}^{\frac{1}{2} N_{\phi}} \sum_{n=0}^{N_{\rho}} \sum_{p=0}^{N_{z}}\left[\widetilde{\psi}_{2 k-1, n p} \cos (k \phi)+\widetilde{\psi}_{2 k, n p} \sin (k \phi)\right] T_{n}(\xi(\rho)) T_{p}(\chi(Z)) .
\end{aligned}
$$

The direct product structure in (53) has been determined by the convention

$$
\widetilde{\boldsymbol{\psi}}\left(m\left(N_{\rho}+1\right)\left(N_{z}+1\right)+n\left(N_{z}+1\right)+p\right)=\widetilde{\psi}_{m n p} .
$$

We now take the matrix representation of the differential operator in (51), and "sparsify" it via multiplication by $\mathcal{B}=B_{\phi}^{2} \otimes B_{\rho[2]}^{2} \otimes B_{Z[2]}^{2}$. In this operator the $B_{\rho[2]}^{2}$ and $B_{Z[2]}^{2}$ correspond to the usual $B$ operators representing integration twice over a coordinate, and leaving two empty rows to be filled by tau-conditions. The operator $B_{\phi}^{2}$ applies to a coordinate with no endpoints, and hence no applicable boundary conditions. It represents double integration over all Fourier modes except the zero mode, which is left unchanged. The matrix that accomplishes this has the explicit form $B_{\phi}^{2}=\operatorname{diag}\left(1,-1,-1,-\frac{1}{4},-\frac{1}{4},-\frac{1}{9},-\frac{1}{9}, \cdots\right)$. Although the operation on $\phi$ does not play a role in handling boundary conditions, it should further enhance the spectrum clustering of the matrix that must be inverted.

With this $\mathcal{B}$ the sparsified form of (53) becomes

$$
\mathcal{B} \mathcal{A}_{\rho}^{2} \boldsymbol{\Delta}=B_{\phi}^{2} \otimes\left(B_{\rho[2]}^{2}+I_{\rho[2]} A_{\rho}^{2}-3 B_{\rho[2]} A_{\rho}\right) \otimes B_{Z[2]}^{2}+I_{\phi[1]} \otimes B_{\rho[2]}^{2} \otimes B_{Z[2]}^{2}+B_{\phi}^{2} \otimes B_{\rho[2]}^{2} A_{\rho}^{2} \otimes I_{Z[2]} .
$$

Our analysis of the terms in the HRWE proportional to $\Omega^{2}$ starts with the expressions

$$
\begin{aligned}
& \rho^{2} \partial_{X}=\rho^{2} \cos \phi \partial_{\rho}-\rho \sin \phi \partial_{\phi} \\
& \rho^{2} \partial_{X}^{2}=\rho^{2} \cos ^{2} \phi \partial_{\rho}^{2}-2 \rho \cos \phi \sin \phi \partial_{\rho} \partial_{\phi}+2 \cos \phi \sin \phi \partial_{\phi}+\rho \sin ^{2} \phi \partial_{\rho}+\sin ^{2} \phi \partial_{\phi}^{2} .
\end{aligned}
$$

With the operator identities $\rho^{2} \partial_{\rho}^{2}=\partial_{\rho}^{2} \rho^{2}-4 \partial_{\rho} \rho+2, \rho \partial_{\rho}=\partial_{\rho} \rho-1$, and $\rho^{2} \partial_{\rho}=\partial_{\rho} \rho^{2}-2 \rho$, we then find

$$
\begin{aligned}
\rho^{2} \partial_{X} & =\partial_{\rho} \rho^{2} \cos \phi-\rho\left(2 \cos \phi+\sin \phi \partial_{\phi}\right) \\
\rho^{2} \partial_{X}^{2} & =\partial_{\rho}^{2} \rho^{2} \cos ^{2} \phi+\partial_{\rho} \rho\left(1-5 \cos ^{2} \phi-2 \cos \phi \sin \phi \partial_{\phi}\right) \\
& +\sin ^{2} \phi \partial_{\phi}^{2}+4 \cos \phi \sin \phi \partial_{\phi}+3 \cos ^{2} \phi-1 .
\end{aligned}
$$


Still viewing these relationships as operator identities, we now exploit the product rule for differentiation to rewrite the terms involving angular derivatives, with the results

$$
\begin{aligned}
& \rho^{2} \partial_{X}=\partial_{\rho} \rho^{2} \cos \phi-\rho\left(\cos \phi+\partial_{\phi} \sin \phi\right) \\
& \rho^{2} \partial_{X}^{2}=\partial_{\rho}^{2} \rho^{2} \cos ^{2} \phi-\partial_{\rho} \rho\left(1+\cos ^{2} \phi+2 \partial_{\phi} \cos \phi \sin \phi\right)+\partial_{\phi}^{2} \sin ^{2} \phi+\sin ^{2} \phi .
\end{aligned}
$$

These equations then yield

$$
\begin{aligned}
\rho^{2} \partial_{X} X & =\partial_{\rho} \rho^{3} \cos ^{2} \phi-\rho^{2}\left(\cos ^{2} \phi+\partial_{\phi} \cos \phi \sin \phi\right) \\
\rho^{2} \partial_{X}^{2} & =\left(\partial_{\rho}^{2} \rho^{2}-3 \partial_{\rho} \rho+1\right) \cos ^{2} \phi \\
& -\partial_{\rho} \rho\left(1-2 \cos ^{2} \phi+2 \partial_{\phi} \cos \phi \sin \phi\right)+\partial_{\phi}^{2} \sin ^{2} \phi+2 \sin ^{2} \phi-1,
\end{aligned}
$$

expressions which prove useful in obtaining the matrix representation of the operator on the righthand side of (51). To optimize the implementation, we have chosen the first term on the righthand side of (64) to match a similar term in the Laplacian part of the operator [cf. Eq. (52)].

We split the HRWE operator on a cylindrical shell as $\rho^{2} L=\rho^{2} \Delta-\Omega^{2} J$, where

$$
J=J_{1}+J_{2}+J_{3}+J_{4}=\underbrace{\rho^{2} \partial_{X} X\left(1-2 \partial_{Z} Z\right)}_{J_{1}}+\underbrace{\rho^{2} \partial_{X}^{2} Z^{2}}_{J_{2}}+\underbrace{\rho^{2} \partial_{Z} Z}_{J_{3}}+\underbrace{\rho^{2} \partial_{Z}^{2} X^{2}}_{J_{4}} .
$$

The piece $\rho^{2} \Delta$ was shown to lead to (57). We now focus on $J$ whose matrix representation stems from the representations of its constituents. Equations (63) and (64) give us

$$
\begin{aligned}
\mathrm{J}_{1} & =C_{\phi}^{2} \otimes D_{\rho} A_{\rho}^{3} \otimes\left(I_{Z}-2 D_{Z} A_{Z}\right)-\left(C_{\phi}^{2}+D_{\phi} C_{\phi} S_{\phi}\right) \otimes A_{\rho}^{2} \otimes\left(I_{Z}-2 D_{Z} A_{Z}\right) \\
\mathrm{J}_{2} & =C_{\phi}^{2} \otimes\left(I+D_{\rho}^{2} A_{\rho}^{2}-3 D_{\rho} A_{\rho}\right) \otimes A_{Z}^{2}-\left(I_{\phi}-2 C_{\phi}^{2}+2 D_{\phi} C_{\phi} S_{\phi}\right) \otimes D_{\rho} A_{\rho} \otimes A_{Z}^{2} \\
& +\left(D_{\phi}^{2} S_{\phi}^{2}+2 S_{\phi}^{2}-1\right) \otimes I_{\rho} \otimes A_{Z}^{2} \\
\mathrm{~J}_{3} & =I_{\phi} \otimes A_{\rho}^{2} \otimes D_{Z} A_{Z} \\
\mathrm{~J}_{4} & =C_{\phi}^{2} \otimes A_{\rho}^{4} \otimes D_{Z}^{2},
\end{aligned}
$$

where $S_{\phi}$ and $C_{\phi}$ are respectively the matrices in the Fourier basis which correspond to multiplication by $\sin \phi$ and $\cos \phi$. Applying the sparsifying matrix $\mathcal{B}=B_{\phi}^{2} \otimes B_{\rho[2]}^{2} \otimes B_{Z[2]}^{2}$, we then have

$$
\begin{aligned}
\mathcal{B J}_{1} & =B_{\phi}^{2} C_{\phi}^{2} \otimes B_{\rho[2]} A_{\rho}^{3} \otimes\left(B_{Z[2]}^{2}-2 B_{Z[2]} A_{Z}\right) \\
& -\left(B_{\phi}^{2} C_{\phi}^{2}+B_{\phi[1]} C_{\phi} S_{\phi}\right) \otimes B_{\rho[2]}^{2} A_{\rho}^{2} \otimes\left(B_{Z[2]}^{2}-2 B_{Z[2]} A_{Z}\right) \\
\mathcal{B} J_{2} & =B_{\phi}^{2} C_{\phi}^{2} \otimes\left(B_{r[2]}^{2}+I_{\rho[2]} A_{\rho}^{2}-3 B_{r[2]} A_{\rho}\right) \otimes B_{Z[2]}^{2} A_{Z}^{2} \\
& -\left(B_{\phi}^{2}-2 B_{\phi}^{2} C_{\phi}^{2}+2 B_{\phi[1]} C_{\phi} S_{\phi}\right) \otimes B_{\rho[2]} A_{\rho} \otimes B_{Z[2]}^{2} A_{Z}^{2} \\
& +\left(I_{\phi[1]} S_{\phi}^{2}+2 B_{\phi}^{2} S_{\phi}^{2}-B_{\phi}^{2}\right) \otimes B_{\rho[2]}^{2} \otimes B_{Z[2]}^{2} A_{Z}^{2} \\
\mathcal{B J}_{3} & =B_{\phi}^{2} \otimes B_{\rho[2]}^{2} A_{\rho}^{2} \otimes B_{Z[2]} A_{Z} \\
\mathcal{B J}_{4} & =B_{\phi}^{2} C_{\phi}^{2} \otimes B_{\rho[2]}^{2} A_{\rho}^{4} \otimes I_{Z[2]} .
\end{aligned}
$$

The sparsified matrix representing (51) is then $\mathcal{B} \mathcal{A}_{\rho}^{2} \mathcal{L}=\mathcal{B A}_{\rho} \Delta-\Omega^{2}\left(\mathcal{B} J_{1}+\mathcal{B J}_{2}+\mathcal{B} J_{3}+\mathcal{B} J_{4}\right)$. 


\begin{tabular}{|l|l|l|}
\hline Boundary & \multicolumn{1}{|c|}{ Rows } & \multicolumn{1}{c|}{ Index restrictions } \\
\hline$\rho=\rho_{\min }$ & $m\left(N_{\rho}+1\right)\left(N_{z}+1\right)+p$ & $0 \leq m \leq N_{\phi}, 2 \leq p \leq N_{z}$ \\
$\rho=\rho_{\max }$ & $m\left(N_{\rho}+1\right)\left(N_{z}+1\right)+\left(N_{z}+1\right)+p$ & $0 \leq m \leq N_{\phi}, 2 \leq p \leq N_{z}$ \\
\hline$Z=Z_{\min }$ & $m\left(N_{\rho}+1\right)\left(N_{z}+1\right)+n\left(N_{z}+1\right)+0$ & $0 \leq n \leq N_{\rho}, 0 \leq m \leq N_{\phi}$ \\
$Z=Z_{\max }$ & $m\left(N_{\rho}+1\right)\left(N_{z}+1\right)+n\left(N_{z}+1\right)+1$ & $0 \leq n \leq N_{\rho}, 0 \leq m \leq N_{\phi}$ \\
\hline
\end{tabular}

TABLE IV. FILLING OF EMPTY ROWS FOR CYLINDERS.

To enforce boundary conditions, we fill empty rows in the matrix $\mathcal{B A}_{\rho} \Delta-\Omega^{2} \mathcal{B}$ J with the tau-conditions. Let $h^{+}(\phi, Z)=\psi\left(\rho_{\max }, \phi, Z\right), h^{-}(\phi, Z)=\psi\left(\rho_{\min }, \phi, Z\right)$ and $f^{+}(\rho, \phi)=$ $\psi\left(\rho, \phi, Z_{\max }\right), f^{-}\left(\rho, \phi, Z_{\min }\right)=\psi\left(\rho, \phi, Z_{\min }\right)$. Then Dirichlet boundary conditions on the inner and outer axial boundaries and on the top and bottom caps are expressible as

$$
\sum_{n=0}^{N_{\rho}} \widetilde{\psi}_{m n p} \delta_{n}^{ \pm}=\tilde{h}_{m p}^{ \pm}, \quad \sum_{p=0}^{N_{z}} \widetilde{\psi}_{m n p} \delta_{p}^{ \pm}=\tilde{f}_{m n}^{ \pm} .
$$

There are $\left(N_{\phi}+1\right)\left(N_{z}+1\right)+\left(N_{\rho}+1\right)\left(N_{\phi}+1\right)$ such equations possible. However, owing to the fact that the caps shares common edges with both the inner and outer axial boundaries, there are $2\left(N_{\phi}+1\right)$ linear dependencies amongst these equations, and in fact the number of available empty rows is precisely

$$
\left(N_{\phi}+1\right)\left(N_{Z}+1\right)+\left(N_{\rho}+1\right)\left(N_{\phi}+1\right)-2\left(N_{\phi}+1\right) .
$$

Table IV shows how we fill zero rows to enforce the boundary conditions.

\section{GLUING OF SUBDOMAINS}

So far we have described individual shell, cylinder, and block subdomains (and their associated tau-conditions) as if they were decoupled. All the subdomains are, of course, coupled and we refer to the process of making them parts of a single problem as "gluing." Matching, or gluing, must be done for each subset of subdomains that touch, whether that touching is a finite volume overlap or a lower-dimensional shared boundary. The global problem requires matching for the following subdomain configurations:

(i) Two adjacent cylinders.

(ii) One inner shell and a combination of cylinders and blocks.

(iii) One cylinder and one block.

(iv) The outer shell and the combination of blocks $B$ and $D$ and all cylinders.

We describe (i) and (ii) in detail, provide a sketch of (iii), and omit a description of (iv) altogether. Although more complicated, a description of (iv) would parallel that of (iii).

Before giving more details, we comment on how such gluing is reflected in the overall linear system. Let, for example, $\widetilde{\boldsymbol{\psi}}^{J}$ and $\widetilde{\boldsymbol{\psi}}^{B}$ respectively represent the vectors of sphericalharmonic Chebyshev and triple Chebyshev expansion coefficients corresponding to the inner shell $J$ and block $B$ of Fig. 1. The overall set of unknowns is the concatenation

$$
\widetilde{\boldsymbol{\Psi}}=\left(\widetilde{\boldsymbol{\psi}}^{J}, \widetilde{\boldsymbol{\psi}}^{H}, \widetilde{\boldsymbol{\psi}}^{B}, \widetilde{\boldsymbol{\psi}}^{C}, \widetilde{\boldsymbol{\psi}}^{D}, \widetilde{\boldsymbol{\psi}}^{1}, \widetilde{\boldsymbol{\psi}}^{2}, \widetilde{\boldsymbol{\psi}}^{3}, \widetilde{\boldsymbol{\psi}}^{4}, \widetilde{\boldsymbol{\psi}}^{5}, \widetilde{\boldsymbol{\psi}}^{O}\right)^{t},
$$


which satisfies a linear system stemming from Eq. (3),

$$
\mathcal{M} \widetilde{\Psi}=\mathcal{B} \widetilde{\mathcal{G}}
$$

where $\widetilde{\mathcal{G}}$ is a similar concatenation of the sources $\widetilde{\mathrm{g}}$ on the individual subdomains. Here $\mathcal{B}$ indicates integration "preconditioning" (sparsification) on all subdomains. Symbolically then, the coefficient matrix $\mathcal{M}$ is $\mathcal{B L}$, now with $\mathcal{L}$ standing for the spectral representation of the HRWE operator $L$ on the whole 2-center domain. In this symbolic view, we have ignored multiplications by radial powers on spheres and cylinders.

Each of the eleven subdomains in Fig. 1 is represented by one of eleven super-blocks $(J-J$, $H-H, \cdots, O-O)$ which sit along the diagonal of the overall super-matrix $\mathcal{M}$ representing the PDE on the whole 2-center domain. We use the term "super-block" here since the matrix corresponding to each subdomain arises, as we have seen, from a direct product structure (and so could be viewed as already in a block form). The supplementary equations needed for gluing are placed within existing empty rows in the same manner as for the tau-conditions. However, the gluing conditions stretch beyond the super-block diagonal, since they are linear relationships between the spectral expansion coefficients on two (or more) separate subdomains. For example, the gluing together of cylinders 1 and 2 (which share a common cap) involves not only filling rows within the 1-1 and 2-2 super-blocks along the diagonal of $\mathcal{M}$, but also filling rows within the 1-2 and 2-1 off-diagonal super-blocks.

\section{A. Gluing of cylinders to cylinders}

As a specific example, let us consider the gluing of cylinders 1 and 2 in Fig. 1, which as indicated share the cap $Z=Z_{*}$, where $Z_{*}$ is $Z_{\max }$ for cylinder 1 and $Z_{\min }$ for cylinder 2 (the common cap has a hole in the middle, since 1 and 2 are cylindrical shells). Let, for example, $\mathcal{P} \psi^{1}$ be shorthand for the numerical solution $\mathcal{P}_{N_{\rho}^{1}, N_{\phi}^{1}, N_{Z}^{1}} \psi^{1}$ for cylinder 1 , as expressed in (55)). The restriction $\mathcal{P} \psi^{1}\left(\rho, \phi, Z_{*}\right)$ is a two-variable function on the cap $Z=Z_{*}$, and it can be expanded in a finite Fourier-Chebyshev series, with $\sum_{k=0}^{N_{Z}^{1}} \widetilde{\psi}_{q n k}^{1} \delta_{k}^{+}$as the corresponding twoindex modal coefficients. Likewise, the restriction $\left(d \mathcal{P} \psi^{1} / d Z\right)\left(\rho, \phi, Z_{*}\right)$ of the $Z$-derivative has a Fourier-Chebyshev series with two-index modal coefficients $\sum_{k=0}^{N_{Z}^{1}} \widetilde{\psi}_{q n k}^{1} \alpha_{1} \nu_{k}^{+}$. The $\alpha_{1}$ factor is a scaling of the Neumann vector $\nu^{+}$, and its presence is necessary since the range of $Z$ is not $[-1,1]$ (details are given in [6]).

On the $Z=Z_{*}$ cap we likewise consider the numerical solution $\mathcal{P} \psi^{2}\left(\rho, \phi, Z_{*}\right)$ and its $Z$-derivative $\left(d \mathcal{P} \psi^{2} / d Z\right)\left(\rho, \phi, Z_{*}\right)$, as determined by the numerical solution $\mathcal{P} \psi^{2}$ on cylinder 2. We distinguish between two cases: (i) both the $N_{\rho}$ and $N_{\phi}$ truncations are the same for cylinders 1 and 2 (but $N_{Z}^{1} \neq N_{Z}^{2}$ is allowed), and (ii) at least one of these truncations differs between the two cylinders (i.e. either $N_{\rho}^{1} \neq N_{\rho}^{2}$ or $N_{\phi}^{1} \neq N_{\phi}^{2}$, or both, hold). Let us first consider case (i), returning to case (ii) in the next paragraph. For case (i) both $\mathcal{P} \psi^{1}\left(\rho, \phi, Z_{*}\right)$ and $\mathcal{P} \psi^{2}\left(\rho, \phi, Z_{*}\right)$ have two-surface modes which are in one-to-one correspondence, and likewise for the derivatives. Therefore, for this case we enforce 4

$$
\sum_{k=0}^{N_{Z}^{1}} \widetilde{\psi}_{q n k}^{1} \delta_{k}^{+}=\sum_{k=0}^{N_{Z}^{2}} \widetilde{\psi}_{q n k}^{2} \delta_{k}^{-}, \quad \sum_{k=0}^{N_{Z}^{1}} \widetilde{\psi}_{q n k}^{1} \alpha_{1} \nu_{k}^{+}=\sum_{k=0}^{N_{Z}^{2}} \widetilde{\psi}_{q n k}^{2} \alpha_{2} \nu_{k}^{-},
$$

4 We regret an error in the definition of $\nu^{-}$in Ref. 6], Eq. (42). The correct expressions are $\nu^{ \pm}=$ $\left[T_{0}^{\prime}( \pm 1), T_{1}^{\prime}( \pm 1), T_{2}^{\prime}( \pm 1), T_{3}^{\prime}( \pm 1), T_{4}^{\prime}( \pm 1), \cdots\right]=[0,1, \pm 4,9, \pm 16, \cdots]$. In Ref. [6] the righthand side of the second equation of $(69)$ is also off by a sign. 
for each Fourier-Chebyshev index pair $(q, n)$. Here, for case (i), the matching conditions enforce continuity between the finite representations $\mathcal{P} \psi^{1}$ and $\mathcal{P} \psi^{2}$ across the cap, and also continuity between the finite representations $d \mathcal{P} \psi^{1} / d Z$ and $d \mathcal{P} \psi^{2} / d Z$. These matching conditions are reflected in the overall matrix $\mathcal{M}$ as follows. As the super-block corresponding to each of the subdomains 1 and 2 has been sparsified in the described fashion, each has a collection of empty rows which are also empty throughout $\mathcal{M}$. In, say, the empty rows stretching across the 1-1 and 1-2 super-blocks, we insert the first set of conditions given in (76). In the empty rows stretching across the 2-2 and 2-1 super-blocks, we similarly place the Neumann conditions, the second set of conditions given in (76). This filling of empty rows to achieve the required matching consists of relationships of modal coefficients with no reference to any "sources"; they are homogeneous equations.

To better understand the issues which will arise in matching volume-overlapping subdomains, we now consider case (ii), the case in which the cylinders 1 and 2 give rise to a disparate set of surface modes on the $Z=Z_{*}$ cap. In this case, for example, we again have $\sum_{k=0}^{N_{Z}^{1}} \widetilde{\psi}_{q n k}^{1} \delta_{k}^{+}$as the modal coefficients determining $\mathcal{P} \psi^{1}\left(\rho, \phi, Z_{*}\right)$, and $\sum_{k=0}^{N_{Z}^{2}} \widetilde{\psi}_{q n k}^{2} \alpha_{2} \nu_{k}^{-}$as the modal coefficients determining $\left(d \mathcal{P} \psi^{2} / d Z\right)\left(\rho, \phi, Z_{*}\right)$. Now, however, (76) is not applicable. Instead, we now fix [cf. the first equation in (74)]

$$
\sum_{k=0}^{N_{z}^{1}} \widetilde{\psi}_{q n k}^{1} \delta_{k}^{+}=\tilde{f}_{q n}^{+} \quad \tilde{e}_{q n}^{-}=\sum_{k=0}^{N_{Z}^{2}} \widetilde{\psi}_{q n k}^{2} \alpha_{2} \nu_{k}^{-},
$$

where here $\tilde{f}_{q n}^{+}$(for $0 \leq q \leq N_{\phi}^{1}$ and $\left.0 \leq n \leq N_{\rho}^{1}\right)$ and $\tilde{e}_{q n}^{-}\left(\right.$for $0 \leq q \leq N_{\phi}^{2}$ and $0 \leq n \leq N_{\rho}^{2}$ ) are not to be viewed as inhomogeneities, rather as expressions built respectively with the modal coefficients for $\mathcal{P} \psi^{2}\left(\rho, \phi, Z_{*}\right)$ and $\left(d \mathcal{P} \psi^{1} / d Z\right)\left(\rho, \phi, Z_{*}\right)$. Note that, as with Eqs. (76)), these equations have the form "cylinder 1 coefficients = cylinder 2 coefficients".

Let us consider only $\tilde{f}_{q n}^{+}$, since similar comments apply to $\tilde{e}_{q n}^{-}$. First, we start with cylinder 1 and define a Chebyshev-Lobatto/Fourier grid $\left\{\left(\rho_{j}, \phi_{i}\right): 0 \leq j \leq N_{\rho}^{1}, 0 \leq i \leq\right.$ $\left.N_{\phi}^{1}\right\}$ on the $Z=Z_{*}$ cap of cylinder 1 . The use of these points affords a double discrete Fourier-Chebyshev transform, through numerical quadrature, relating function values at the points and mode coefficients. (In practice, we have exploited the trigonometric form of the Chebyshev polynomials and have used the FFT to define both the Fourier and Chebyshev components of this transform.) The double discrete transform allows us to express the modal coefficients $\tilde{f}_{q n}^{+}$in terms of the function values $f_{i j}^{+}$, at $\rho_{i}, \phi_{j}$, for $Z=Z_{*}$ on cylinder 1 , in a form

$$
\tilde{f}_{q n}^{+}=\sum_{i=0}^{N_{\rho}^{1}} \sum_{j=0}^{N_{\phi}^{1}} \mathcal{F}_{q n, i j} f_{i j}^{+} .
$$

Next, at the nodal points $\left(\rho_{j}, \phi_{i}\right)$ of cylinder 1 , we evaluate $f_{i j}^{+}$in terms of the expansion for the solution on cylinder 2 , thereby finding

$$
f_{i j}^{+}=\mathcal{P} \psi^{2}\left(\rho_{j}, \phi_{i}, Z_{*}\right)=\sum_{q=0}^{N_{\phi}^{2}} \sum_{n=0}^{N_{\rho}^{2}} \mathcal{E}_{i j, q n} \sum_{k=0}^{N_{Z}^{2}} \widetilde{\psi}_{q n k}^{2} \delta_{k}^{-} .
$$

Here, the values $\mathcal{E}_{i j, q n}$ arise from the evaluations of the modal functions (Chebyshev and Fourier) of cylinder 2 at the nodal points $\left(\rho_{j}, \phi_{i}\right)$ of cylinder 1 . When the expressions for $f_{i j}^{+}$ from (79) are substituted in (78), we get expressions for $\tilde{f}_{q n}^{+}$in terms of the modal coefficients 
$\widetilde{\psi}_{q n k}^{2}$ representing the solution in cylinder 2. Finally, we substitute this $\tilde{f}_{q n}^{+}$into (77), which yields relationships between the modal coefficients on cylinder 1 and cylinder 2 that express continuity of the solution across $Z=Z_{*}$.

The linear relationships (77) would likewise be inserted into the overall coefficient matrix $\mathcal{M}$. Similar to before, the righthand side of the first equation in (177) would fill empty rows stretching across the 1-2 super-block, with the $\delta^{+}$vectors on the lefthand side filling empty rows across the 1-1 super-block. The relationships expressed in the second equation in (77) would fill empty rows stretching across the 2-2 and 2-1 super-blocks Finally, we note that the equations (77) reduce to (76) when $N_{\phi}^{1}=N_{\phi}^{2}$ and $N_{\rho}^{1}=N_{\rho}^{2}$.

\section{B. Gluing of an inner shell to cylinders and blocks}

The shells $J$ and $H$ depicted in Fig. 1 overlap multiple blocks and cylinders, and for this overlap the issue of gluing is complicated. Since the issue is essentially the same for the gluing of $H$ to blocks $C, D$ and cylinders $3,4,5$ or $J$ to blocks $B, C$ and cylinders $1,2,3$, let us here focus on the first case. The issue here is that parts of the outer boundary $\partial H^{+}$of $H$ sit in blocks $C, D$ and cylinders $3,4,5$. Let \# represent one of the tags $C, D, 3,4,5$, and let us consider the portion $\partial H_{\#}^{+}$of $\partial H^{+}$which intersects subdomain \#. At nodal points on $\partial H_{\#}^{+}$we require that the values of $\psi$ agree whether they are computed with the spectral representation $\widetilde{\boldsymbol{\psi}}^{H}$ for $H$ or the spectral representation $\widetilde{\boldsymbol{\psi}}^{\#}$ for $\#$. For nodal points $\left(\theta_{j}, \phi_{k}\right)$ this condition is [cf. Eq. (50)]

$$
h_{j k}^{+} \equiv h^{+}\left(\theta_{j}, \phi_{k}\right)=\mathcal{P} \psi^{\#}\left(\boldsymbol{x}\left(r_{\max }, \theta_{k}, \phi_{j}\right)\right) \text { for }\left(r_{\max }, \theta_{j}, \phi_{k}\right) \in \partial H_{\#}^{+} \text {. }
$$

Here $\mathcal{P} \psi^{\#}$ is the numerical solution ( $\mathcal{P}$ indicates finite expansion) associated with $\widetilde{\boldsymbol{\psi}} \#$, and $\boldsymbol{x}$ are the relevant $3 \mathrm{D}$ coordinates on subdomain \#. Looping over all of the subdomains $\#=C, D, 3,4,5$ defines the grid function $h_{j k}^{+}$at all nodal points of $\partial H_{\#}^{+}$. The explicit matching conditions (equivalent to the + case in (50) ) can then be realized by expressing the spherical harmonic transform $\tilde{h}_{\ell q}^{+}=\sum_{j=0}^{N_{\theta}} \sum_{k=0}^{2 N_{\theta}} \mathcal{S}_{\ell q, j k} h_{j k}^{+}$as a matrix-vector product involving all $\widetilde{\boldsymbol{\psi}}^{\#}$. The resulting equations are placed within empty rows of $\mathcal{M}$ which stretch across the $H$ - $H$ and $H$-\# super-blocks.

Again let \# represent one of the tags $C, D, 3,4,5$. Then the boundary $\partial \#$ of subdomain \# includes a portion $\partial \#_{H}$ overlapping shell $H$ which gives rise to further gluing relations. These equations will be inserted into empty rows of $\mathcal{M}$ which stretch across the \#-\# and $\#-H$ super-blocks. For concreteness, we consider only the $\#=C$ case. Here the + case of (12) is relevant, although the $\tilde{h}_{n m}^{+}$now arise as the double $X Y$-Chebyshev transform of

$$
\begin{aligned}
h_{j k}^{+} & =\sum_{n=0}^{N_{r}} \sum_{\ell=0}^{N_{\theta}} \widetilde{\psi}_{\ell 0 n}^{H} \bar{P}_{\ell 0}\left(\cos \theta_{j k}\right) T_{n}\left(\xi\left(r_{j k}\right)\right) \\
& +\sum_{n=0}^{N_{r}} \sum_{\ell=1}^{N_{\theta}} \sum_{m=1}^{N_{\theta}} \bar{P}_{\ell m}\left(\cos \theta_{j k}\right)\left[\widetilde{\psi}_{\ell, 2 m-1, n}^{H} \cos \left(m \phi_{j k}\right)+\widetilde{\psi}_{\ell, 2 m, n}^{H} \sin \left(m \phi_{j k}\right)\right] T_{n}\left(\xi\left(r_{j k}\right)\right) .
\end{aligned}
$$

The $H$ point $\left(r_{j k}, \theta_{j k}, \phi_{j k}\right)$ corresponds to a Chebyshev-Gauss-Lobatto collocation point $\left(X\left(\xi_{j}\right), Y\left(\eta_{k}\right), Z_{\max }\right)$ along the top $X Y$-face of block $C$. Again, via a matrix representation $\widetilde{h}_{n m}^{+}=\sum_{j=0}^{N_{X}} \sum_{k=0}^{N_{Y}} \mathcal{F}_{n m, j k} h_{j k}^{+}$of the transform, we may express this matching condition more 
directly. As mentioned, these equations will be inserted into empty rows of $\mathcal{M}$ which stretch across the $C-C$ and $C$ - $H$ super-blocks.

\section{Gluing of a cylinder to a block}

Here we sketch either the gluing of block $B$ and cylinder 1 , block $C$ and cylinder 3 , or block $D$ and cylinder 5 . We focus on the middle case as a representative example. This process involves both (a) gluing two $Y Z$ and two $X Z$ faces of block $C$ to cylinder 3, and (b) gluing the inner radial boundary of the cylinder to the block. The process for (a) is similar to the gluing described in the last paragraph (in which a face of $C$ is glued to $H$ ), and we omit a description. To express the matching equations which enforce (b), we first define

$$
q_{j k}^{-}=\sum_{n=0}^{N_{X}} \sum_{m=0}^{N_{Y}} \sum_{p=0}^{N_{Z}} \widetilde{\psi}_{n m p}^{C} T_{n}\left(\xi\left(X_{j k}\right)\right) T_{m}\left(\eta\left(Y_{j k}\right)\right) T_{p}\left(\chi\left(Z_{j k}\right)\right) .
$$

Here we use the following points:

$$
\left(X_{j k}, Y_{j k}, Z_{j k}\right)=\left(X\left(\rho_{\min }, \phi_{j}, z_{k}\right), Y\left(\rho_{\min }, \phi_{j}, z_{k}\right), Z\left(\rho_{\min }, \phi_{j}, z_{k}\right)\right),
$$

where $\left(\rho_{\min }, \phi_{j}, z_{k}\right)$ are nodal points along the inner radial boundary of cylinder 3 . Next, we consider the Fourier-Chebyshev transform $\tilde{q}_{m p}^{-}=\sum_{j=0}^{N_{\phi}} \sum_{k=0}^{N_{z}} \mathcal{C}_{m p, j k} q_{j k}^{-}$. In terms of the transform the matching equations are

$$
\sum_{n=0}^{N_{\rho}} \widetilde{\psi}_{m n p}^{3} \delta_{n}^{-}=\tilde{q}_{m p}^{-}
$$

These equations are inserted into empty rows of $\mathcal{M}$ which stretch across the 3-3 and 3-C superblocks.

\section{NUMERICAL SOLUTION OF THE 3D HRWE}

Both on single subdomains and on the global 2-center multidomain $\mathcal{D}$, this section considers numerical solution of the HRWE for the field of two point sources in a circular binary orbit. For this problem we have an essentially closed-form exact solution, a superposition of the fields for two point sources, each point source in a circular orbit and described by the Liénard-Wiechert solution (A11) found in the appendix. A numerical solution is a collection of modal expansion coefficients; however, comparisons with the exact solution are always computed in physical space on the nodal grid (or grids in the multidomain case) dual to the modal expansion 5 All numerical solves are performed iteratively with preconditioned GMRES [35], and this section also describes the relevant preconditioning (both for subdomain solves and for the global multidomain solve).

Using the sparse representations described in Sec. II, in Sec. IV A we numerically solve the HRWE on the following subdomains (cf. Fig. 1 and Table【): the outer shell $O$, (inner) spherical shell $J$, (inner) cylindrical shell 5 , and (inner) block $D$. For each subdomain labeled (inner) the HRWE operator is implemented as a matrix-vector multiply within preconditioned

\footnotetext{
${ }^{5}$ As these nodal grids are coarse, the $L_{2}$ and $L_{\infty}$ norms reported in the tables do not settle down quickly.
} 


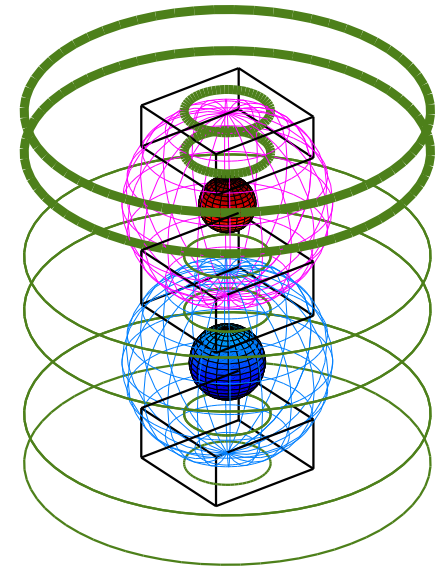

(a) Cylinder experiment.

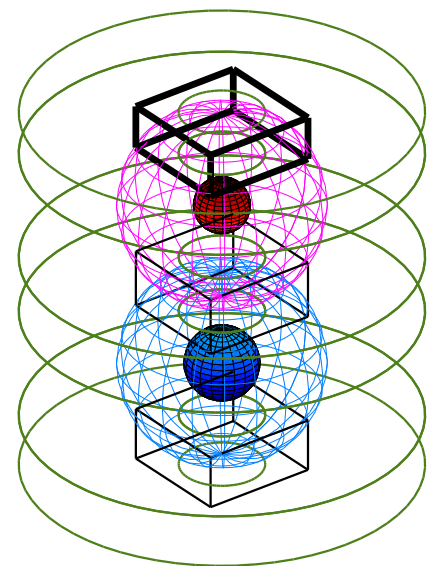

(b) Block experiment.

FIG. 2. INDIVIDUAL SUBDOMAIns. We consider cylindrical shell 5 highlighted in the left figure, block D highlighted in the right, and the bottom inner spherical shell J shown in both.

GMRES without restarts. For these subdomain solves, Dirichlet boundary conditions are taken from the exact Liénard-Wiechert solution, but the outer shell also problem involves the radiation boundary conditions given in Eq. (25). The particular subdomains considered in Sec. IVA are representative, and similar experimentation on each subdomain has determined the chosen truncations for the 2-center multidomain tests described in Sec. IVB, Such experiments empirically yield appropriate truncations necessary to achieve a desired accuracy. All tests in Secs. IVA and IVB involve the following configuration: two charges, one with $z_{H}=1, Q_{H}=1$ and the other with $z_{J}=-0.9, Q_{J}=0.5$. Sec. IVA considers $\Omega=0.1,0.3,0.5,0.7$. The rotation rate $\Omega=0.3$ is large for an astrophysical problem, while $\Omega=0.5,0.7$ are very large rates chosen to "break" our numerical methods.

\section{A. Numerical solution on individual subdomains}

We consider the outer shell first, since results for this subdomain are the most disappointing. The solve for this subdomain differs from the rest. Indeed, since the representation of the HRWE on the outer shell is comprised of $(\ell, m)$ blocks along the block diagonal, we invert each of these physical modes using $L U$-factorization [the "physical modes" are those not annihilated by the projection operator $\mathbb{P}$ defined in (17)]. Let $N_{\theta}=\ell_{\max }$, so that $\mathcal{N}=\left(N_{\theta}+1\right)\left(2 N_{\theta}+1\right)\left(N_{r}+1\right)$ is the system size, with $\mathcal{N}^{2} \sim 4 N_{\theta}^{4} N_{r}^{2}$ the storage requirement for the full coefficient matrix. However, storage of all blocks involves $\left(N_{\theta}+1\right)$ matrices of size $\left(N_{r}+1\right)$-by- $\left(N_{r}+1\right)$, one for each zero mode, in addition to $\frac{1}{2} N_{\theta}\left(N_{\theta}+1\right)$ matrices of size $2\left(N_{r}+1\right)$-by-2 $\left(N_{r}+1\right)$, one for each fixed- $m \cos / \sin$ pair. Therefore, storage for this solve scales like

$$
\left(N_{\theta}+1\right)\left(N_{r}+1\right)^{2}+2 N_{\theta}\left(N_{\theta}+1\right)\left(N_{r}+1\right)^{2} \sim 2 N_{\theta}^{2} N_{r}^{2}=O\left(N_{r} \cdot \mathcal{N}\right) .
$$

Table $\mathrm{V}$ collects results for the outer shell experiment. While excellent for $\Omega=0.1$, they exhibit marked degradation as $\Omega$ increases. A splitting of the single outer shell into multiple concentric shells would likely yield improved accuracy for large $\Omega$. 


\begin{tabular}{|c|c|c|c|c|c|}
\hline \multicolumn{6}{|c|}{$\Omega=0.1$} \\
\hline \hline$N_{r}$ & $\ell_{\max }$ & $L_{2}$ error & $L_{2}$ norm & $L_{\infty}$ error & $L_{\infty}$ norm \\
\hline 65 & 10 & $1.1899 \mathrm{E}-05$ & $1.8977 \mathrm{E}-01$ & $2.7970 \mathrm{E}-04$ & $1.1808 \mathrm{E}+00$ \\
85 & 18 & $2.1320 \mathrm{E}-07$ & $1.8725 \mathrm{E}-01$ & $3.6548 \mathrm{E}-06$ & $1.1810 \mathrm{E}+00$ \\
125 & 28 & $2.4580 \mathrm{E}-10$ & $1.8472 \mathrm{E}-01$ & $5.4193 \mathrm{E}-09$ & $1.1810 \mathrm{E}+00$ \\
185 & 42 & $2.2846 \mathrm{E}-13$ & $1.8297 \mathrm{E}-01$ & $2.7858 \mathrm{E}-12$ & $1.1810 \mathrm{E}+00$ \\
\hline
\end{tabular}

\begin{tabular}{|c|c|c|c|c|c|}
\hline \multicolumn{7}{|c|}{$\Omega=0.3$} \\
\hline \hline$N_{r}$ & $\ell_{\max }$ & $L_{2}$ error & $L_{2}$ norm & $L_{\infty}$ error & $L_{\infty}$ norm \\
\hline 65 & 10 & $7.4142 \mathrm{E}-04$ & $1.9030 \mathrm{E}-01$ & $1.0557 \mathrm{E}-02$ & $1.2624 \mathrm{E}+00$ \\
85 & 18 & $9.4481 \mathrm{E}-04$ & $1.8777 \mathrm{E}-01$ & $1.3180 \mathrm{E}-02$ & $1.2628 \mathrm{E}+00$ \\
125 & 28 & $1.2701 \mathrm{E}-04$ & $1.8523 \mathrm{E}-01$ & $2.0861 \mathrm{E}-03$ & $1.2628 \mathrm{E}+00$ \\
185 & 42 & $5.1221 \mathrm{E}-06$ & $1.8347 \mathrm{E}-01$ & $1.2307 \mathrm{E}-04$ & $1.2628 \mathrm{E}+00$ \\
\hline
\end{tabular}

\begin{tabular}{|c|c|c|c|c|c|}
\hline \multicolumn{7}{|c|}{$\Omega=0.5$} \\
\hline \hline$N_{r}$ & $\ell_{\max }$ & $L_{2}$ error & $L_{2}$ norm & $L_{\infty}$ error & $L_{\infty}$ norm \\
\hline 65 & 10 & $1.4622 \mathrm{E}-02$ & $1.9196 \mathrm{E}-01$ & $1.9489 \mathrm{E}-01$ & $1.4875 \mathrm{E}+00$ \\
85 & 18 & $5.6234 \mathrm{E}-02$ & $1.9726 \mathrm{E}-01$ & $7.8285 \mathrm{E}-01$ & $1.4904 \mathrm{E}+00$ \\
125 & 28 & $6.7047 \mathrm{E}-03$ & $1.8689 \mathrm{E}-01$ & $1.4751 \mathrm{E}-01$ & $1.4996 \mathrm{E}+00$ \\
185 & 42 & $3.6457 \mathrm{E}-03$ & $1.8503 \mathrm{E}-01$ & $7.6831 \mathrm{E}-02$ & $1.5055 \mathrm{E}+00$ \\
\hline
\end{tabular}

\begin{tabular}{|c|c|c|c|c|c|}
\hline \multicolumn{7}{|c|}{$\Omega=0.7$} \\
\hline \hline$N_{r}$ & $\ell_{\max }$ & $L_{2}$ error & $L_{2}$ norm & $L_{\infty}$ error & $L_{\infty}$ norm \\
\hline 65 & 10 & $1.8330 \mathrm{E}-01$ & $2.6488 \mathrm{E}-01$ & $4.2561 \mathrm{E}+00$ & $4.9750 \mathrm{E}+00$ \\
85 & 18 & $3.8033 \mathrm{E}-02$ & $1.9502 \mathrm{E}-01$ & $7.1574 \mathrm{E}-01$ & $2.0804 \mathrm{E}+00$ \\
125 & 28 & $3.9821 \mathrm{E}-02$ & $1.9408 \mathrm{E}-01$ & $1.0589 \mathrm{E}+00$ & $2.2504 \mathrm{E}+00$ \\
185 & 42 & $2.7380 \mathrm{E}-02$ & $1.9029 \mathrm{E}-01$ & $7.3279 \mathrm{E}-01$ & $2.2182 \mathrm{E}+00$ \\
\hline
\end{tabular}

TABLE V. OUTER SPHERICAL SHELL $O$ TEST

We next consider the inner spherical shell $J$. Note that $\Omega=0.5$ corresponds to a shell just within the elliptic region, but $\Omega=0.7$ corresponds to a shell which does not lie fully within the elliptic region. Tables VI and VII list errors, without and with preconditioning. For the sake of comparison, in both these and subsequent tables we have chosen the same requested tolerances (for the GMRES solve) uniformly in $\Omega$, although for larger $\Omega$ the achieved accuracy could likely be attained with a weaker tolerance and fewer iterations. The chosen preconditioner is block-Jacobi. Namely, we invert physical $(\ell, m)$ modes along the block diagonal using a precomputed $L U$-factorization. The storage and scaling properties for this preconditioner are exactly the same as described for the direct solve on the outer shell. However, for inner shells the HRWE representation is not block diagonal in $(\ell, m)$ pairs (as on the outer shell), rather the operator has significant bandwidth in both indices. Therefore, storage of the full matrix for an inner shell would require correspondingly larger memory relative to the preconditioner storage. Further, the preconditioner storage requirement could be reduced by inverting each sin/cos block mode independently. Moreover, were the preconditioner chosen to correspond to only the Laplacian part of the operator, then it could be used for the solves on both shells if their dimensions and truncations were the same. In any case, the chosen preconditioner notably improves the convergence of the GMRES solver.

Table VIII list the results for the corresponding single cylinder experiment, with block $L U$-preconditioning similar to before. That is, for each Fourier mode we invert the associated diagonal block. Our choice (56) of direct product structure for the cylinders determines that each block is $\left(N_{\rho}+1\right)\left(N_{Z}+1\right)$-by- $\left(N_{\rho}+1\right)\left(N_{Z}+1\right)$. For cylinders, preconditioning 


\begin{tabular}{|c|c|c|c|c|c|c|c|}
\hline \multicolumn{2}{|c|}{$\Omega=0.1$} \\
\hline \hline$N_{r}$ & $\ell_{\max }$ & $L_{2}$ error & $L_{2}$ norm & $L_{\infty}$ error & $L_{\infty}$ norm & iterations & tolerance \\
\hline 12 & 12 & $3.4702 \mathrm{E}-06$ & $1.3516 \mathrm{E}+00$ & $2.2656 \mathrm{E}-05$ & $1.9143 \mathrm{E}+00$ & 54 & $1.0000 \mathrm{E}-07$ \\
18 & 23 & $9.9814 \mathrm{E}-09$ & $1.3499 \mathrm{E}+00$ & $3.6488 \mathrm{E}-08$ & $1.9168 \mathrm{E}+00$ & 129 & $1.0000 \mathrm{E}-09$ \\
20 & 33 & $6.0107 \mathrm{E}-11$ & $1.3498 \mathrm{E}+00$ & $2.6944 \mathrm{E}-10$ & $1.9173 \mathrm{E}+00$ & 238 & $1.0000 \mathrm{E}-11$ \\
30 & 46 & $6.2864 \mathrm{E}-13$ & $1.3481 \mathrm{E}+00$ & $2.8333 \mathrm{E}-12$ & $1.9176 \mathrm{E}+00$ & 415 & $1.0000 \mathrm{E}-13$ \\
\hline
\end{tabular}

\begin{tabular}{|c|c|c|c|c|c|c|c|}
\hline \multicolumn{2}{|c|}{$\Omega=0.3$} \\
\hline \hline$N_{r}$ & $\ell_{\max }$ & $L_{2}$ error & $L_{2}$ norm & $L_{\infty}$ error & $L_{\infty}$ norm & iterations & tolerance \\
\hline 12 & 12 & $7.6593 \mathrm{E}-06$ & $1.3566 \mathrm{E}+00$ & $7.8541 \mathrm{E}-05$ & $1.9255 \mathrm{E}+00$ & 57 & $1.0000 \mathrm{E}-07$ \\
18 & 23 & $2.1272 \mathrm{E}-08$ & $1.3550 \mathrm{E}+00$ & $3.8188 \mathrm{E}-07$ & $1.9278 \mathrm{E}+00$ & 138 & $1.0000 \mathrm{E}-09$ \\
20 & 33 & $1.1806 \mathrm{E}-10$ & $1.3550 \mathrm{E}+00$ & $2.4707 \mathrm{E}-09$ & $1.9283 \mathrm{E}+00$ & 255 & $1.0000 \mathrm{E}-11$ \\
30 & 46 & $4.3184 \mathrm{E}-13$ & $1.3532 \mathrm{E}+00$ & $5.2289 \mathrm{E}-12$ & $1.9286 \mathrm{E}+00$ & 442 & $1.0000 \mathrm{E}-13$ \\
\hline
\end{tabular}

\begin{tabular}{|c|c|c|c|c|c|c|c|}
\hline \multicolumn{2}{|c|}{$\Omega=0.5$} \\
\hline \hline$N_{r}$ & $\ell_{\max }$ & $L_{2}$ error & $L_{2}$ norm & $L_{\infty}$ error & $L_{\infty}$ norm & iterations & tolerance \\
\hline 12 & 12 & $2.3588 \mathrm{E}-05$ & $1.3782 \mathrm{E}+00$ & $2.7337 \mathrm{E}-04$ & $1.9561 \mathrm{E}+00$ & 61 & $1.0000 \mathrm{E}-07$ \\
18 & 23 & $8.3249 \mathrm{E}-08$ & $1.3769 \mathrm{E}+00$ & $1.5539 \mathrm{E}-06$ & $1.9579 \mathrm{E}+00$ & 146 & $1.0000 \mathrm{E}-09$ \\
20 & 33 & $4.9181 \mathrm{E}-10$ & $1.3770 \mathrm{E}+00$ & $1.1765 \mathrm{E}-08$ & $1.9583 \mathrm{E}+00$ & 271 & $1.0000 \mathrm{E}-11$ \\
30 & 46 & $7.9161 \mathrm{E}-13$ & $1.3752 \mathrm{E}+00$ & $1.8530 \mathrm{E}-11$ & $1.9585 \mathrm{E}+00$ & 471 & $1.0000 \mathrm{E}-13$ \\
\hline
\end{tabular}

\begin{tabular}{|c|c|c|c|c|c|c|c|}
\hline \multicolumn{2}{|c|}{$\Omega=0.7$} \\
\hline \hline$N_{r}$ & $\ell_{\max }$ & $L_{2}$ error & $L_{2}$ norm & $L_{\infty}$ error & $L_{\infty}$ norm & iterations & tolerance \\
\hline 12 & 12 & $4.7252 \mathrm{E}-04$ & $1.4267 \mathrm{E}+00$ & $3.1710 \mathrm{E}-03$ & $2.2326 \mathrm{E}+00$ & 87 & $1.0000 \mathrm{E}-07$ \\
18 & 23 & $1.1589 \mathrm{E}-05$ & $1.4259 \mathrm{E}+00$ & $1.2079 \mathrm{E}-04$ & $2.2356 \mathrm{E}+00$ & 461 & $1.0000 \mathrm{E}-09$ \\
20 & 33 & $8.3186 \mathrm{E}-08$ & $1.4262 \mathrm{E}+00$ & $6.8077 \mathrm{E}-07$ & $2.2353 \mathrm{E}+00$ & 1459 & $1.0000 \mathrm{E}-11$ \\
30 & 46 & $1.3484 \mathrm{E}-09$ & $1.4243 \mathrm{E}+00$ & $1.4001 \mathrm{E}-08$ & $2.2351 \mathrm{E}+00$ & 5215 & $1.0000 \mathrm{E}-13$ \\
\hline
\end{tabular}

TABLE VI. INNER SPHERICAL SHELL TEST $J$ WITHOUT PRECONDITIONING.

amounts to direct inversion of each Fourier mode along the block diagonal. With $\mathcal{N}=$ $\left(N_{\phi}+1\right)\left(N_{\rho}+1\right)\left(N_{z}+1\right)$ the system size, the storage requirement for the preconditioner requires $N_{\phi}+1$ matrices of size $\left(N_{\rho}+1\right)\left(N_{z}+1\right)$-by- $\left(N_{\rho}+1\right)\left(N_{z}+1\right)$, and so scales like so

$$
\left(N_{\rho}+1\right)^{2}\left(N_{z}+1\right)^{2}\left(N_{\phi}+1\right)=O\left(N_{\rho} N_{z} \cdot \mathcal{N}\right) .
$$

While $N_{\rho} N_{z} \mathcal{N}<\mathcal{N}^{2}$, this requirement is somewhat memory intensive. However, we have observed essentially the same performance when using the corresponding Laplacian part of the operator to define the preconditioner. Provided that the dimensions and truncations of two individual cylinders match, the same preconditioner could then be used for both.

Table IX list errors for the block experiment, and again with a block-Jacobi preconditioner. In this case there are $N_{x}+1$ blocks with size $\left(N_{y}+1\right)\left(N_{z}+1\right)$-by- $\left(N_{y}+1\right)\left(N_{z}+1\right)$. Storage of the block preconditioner therefore scales as

$$
\left(N_{x}+1\right)\left(N_{y}+1\right)^{2}\left(N_{z}+1\right)^{2}=O\left(N_{y} N_{z} \cdot \mathcal{N}\right) .
$$

Again, were the preconditioner based on the Laplacian part of the operator, it might be reused for the solves on different blocks.

\section{B. Numerical solution on the 2-center multidomain}

We have also used GMRES [35] to solve the linear system $\mathcal{M} \widetilde{\Psi}=\mathcal{B} \widetilde{\mathcal{G}}$ given in Eq. (75) and corresponding to the HRWE on the full 2-center multidomain $\mathcal{D}$. Section II has described 


\begin{tabular}{|c|c|c|c|c|c|c|c|}
\hline \multicolumn{2}{|c|}{$\Omega=0.1$} \\
\hline \hline$N_{r}$ & $\ell_{\max }$ & $L_{2}$ error & $L_{2}$ norm & $L_{\infty}$ error & $L_{\infty}$ norm & iterations & tolerance \\
\hline 12 & 12 & $3.4196 \mathrm{E}-06$ & $1.3516 \mathrm{E}+00$ & $2.2499 \mathrm{E}-05$ & $1.9143 \mathrm{E}+00$ & 3 & $1.0000 \mathrm{E}-07$ \\
18 & 23 & $3.4877 \mathrm{E}-09$ & $1.3499 \mathrm{E}+00$ & $3.6560 \mathrm{E}-08$ & $1.9168 \mathrm{E}+00$ & 4 & $1.0000 \mathrm{E}-09$ \\
20 & 33 & $1.3949 \mathrm{E}-11$ & $1.3498 \mathrm{E}+00$ & $2.1394 \mathrm{E}-10$ & $1.9173 \mathrm{E}+00$ & 4 & $1.0000 \mathrm{E}-11$ \\
30 & 46 & $3.0104 \mathrm{E}-14$ & $1.3481 \mathrm{E}+00$ & $3.0975 \mathrm{E}-13$ & $1.9176 \mathrm{E}+00$ & 5 & $1.0000 \mathrm{E}-13$ \\
\hline
\end{tabular}

\begin{tabular}{|c|c|c|c|c|c|c|c|}
\hline \multicolumn{2}{|c|}{$\Omega=0.3$} \\
\hline \hline$N_{r}$ & $\ell_{\max }$ & $L_{2}$ error & $L_{2}$ norm & $L_{\infty}$ error & $L_{\infty}$ norm & iterations & tolerance \\
\hline 12 & 12 & $7.6487 \mathrm{E}-06$ & $1.3566 \mathrm{E}+00$ & $7.8536 \mathrm{E}-05$ & $1.9255 \mathrm{E}+00$ & 4 & $1.0000 \mathrm{E}-07$ \\
18 & 23 & $2.0798 \mathrm{E}-08$ & $1.3550 \mathrm{E}+00$ & $3.8215 \mathrm{E}-07$ & $1.9278 \mathrm{E}+00$ & 6 & $1.0000 \mathrm{E}-09$ \\
20 & 33 & $1.0362 \mathrm{E}-10$ & $1.3550 \mathrm{E}+00$ & $2.4686 \mathrm{E}-09$ & $1.9283 \mathrm{E}+00$ & 7 & $1.0000 \mathrm{E}-11$ \\
30 & 46 & $1.0361 \mathrm{E}-13$ & $1.3532 \mathrm{E}+00$ & $3.1604 \mathrm{E}-12$ & $1.9286 \mathrm{E}+00$ & 9 & $1.0000 \mathrm{E}-13$ \\
\hline
\end{tabular}

\begin{tabular}{|c|c|c|c|c|c|c|c|}
\hline \multicolumn{2}{|c|}{$\Omega=0.5$} \\
\hline \hline$N_{r}$ & $\ell_{\max }$ & $L_{2}$ error & $L_{2}$ norm & $L_{\infty}$ error & $L_{\infty}$ norm & iterations & tolerance \\
\hline 12 & 12 & $2.3577 \mathrm{E}-05$ & $1.3782 \mathrm{E}+00$ & $2.7330 \mathrm{E}-04$ & $1.9561 \mathrm{E}+00$ & 7 & $1.0000 \mathrm{E}-07$ \\
18 & 23 & $8.3163 \mathrm{E}-08$ & $1.3769 \mathrm{E}+00$ & $1.5545 \mathrm{E}-06$ & $1.9579 \mathrm{E}+00$ & 10 & $1.0000 \mathrm{E}-09$ \\
20 & 33 & $4.9053 \mathrm{E}-10$ & $1.3770 \mathrm{E}+00$ & $1.1755 \mathrm{E}-08$ & $1.9583 \mathrm{E}+00$ & 13 & $1.0000 \mathrm{E}-11$ \\
30 & 46 & $6.2389 \mathrm{E}-13$ & $1.3752 \mathrm{E}+00$ & $1.8454 \mathrm{E}-11$ & $1.9585 \mathrm{E}+00$ & 17 & $1.0000 \mathrm{E}-13$ \\
\hline
\end{tabular}

\begin{tabular}{|c|c|c|c|c|c|c|c|}
\hline \multicolumn{2}{|c|}{$\Omega=0.7$} \\
\hline \hline$N_{r}$ & $\ell_{\max }$ & $L_{2}$ error & $L_{2}$ norm & $L_{\infty}$ error & $L_{\infty}$ norm & iterations & tolerance \\
\hline 12 & 12 & $4.7262 \mathrm{E}-04$ & $1.4267 \mathrm{E}+00$ & $3.1713 \mathrm{E}-03$ & $2.2326 \mathrm{E}+00$ & 30 & $1.0000 \mathrm{E}-07$ \\
18 & 23 & $1.1596 \mathrm{E}-05$ & $1.4259 \mathrm{E}+00$ & $1.2093 \mathrm{E}-04$ & $2.2356 \mathrm{E}+00$ & 154 & $1.0000 \mathrm{E}-09$ \\
20 & 33 & $8.3367 \mathrm{E}-08$ & $1.4262 \mathrm{E}+00$ & $6.8017 \mathrm{E}-07$ & $2.2353 \mathrm{E}+00$ & 429 & $1.0000 \mathrm{E}-11$ \\
30 & 46 & $1.3430 \mathrm{E}-09$ & $1.4243 \mathrm{E}+00$ & $1.3999 \mathrm{E}-08$ & $2.2351 \mathrm{E}+00$ & 1390 & $1.0000 \mathrm{E}-13$ \\
\hline
\end{tabular}

TABLE VII. INNER SPHERICAL SHELL $J$ TEST WITH PRECONDITIONING.

the coefficient matrix $\mathcal{M}$, and therefore also implementation of the "matrix-vector multiply" $\widetilde{\Psi} \rightarrow \mathcal{M} \widetilde{\Psi}$. Implementation of this multiply is required by the GMRES algorithm (with or without preconditioning). However, a simple unpreconditioned GMRES strategy results in extremely poor convergence. Therefore, we have implemented (left) preconditioned GMRES which further requires implementation of the operation $\widetilde{\Psi} \rightarrow \mathcal{M}_{\text {approx }}^{-1} \widetilde{\Psi}$ in terms of a suitable approximate inverse $\mathcal{M}_{\text {approx }}^{-1} \simeq \mathcal{M}^{-1}$. In this section we describe application of $\mathcal{M}_{\text {approx }}^{-1}$, and document tests of the full global solve. We stress that the preconditioning afforded by $\mathcal{M}_{\text {approx }}^{-1}$ is neither (i) the integration "preconditioning" technique used to achieve sparse representations of (44) on each of the basic subdomains nor (ii) the preconditioning (typically a form of block- $L U$ ) used for individual subdomain solves. However, type (ii) preconditioning does define part of the $\mathcal{M}_{\text {approx }}^{-1}$ application.

The action of $\mathcal{M}_{\text {approx }}^{-1}$ is defined through the simple alternating Schwarz method [42]. Application of this preconditioner relies on independent numerical solves over (i) the inner shells $J$ and $H$, (ii) the glued subregion 6 comprised of blocks and cylinders depicted in Fig. 3, and (iii) the outer spherical shell $O$. More precisely, starting with a vanishing initial vector $\widetilde{\boldsymbol{\Psi}}$ we perform the following iteration.

1. Solve (also by GMRES, as described in Sec. IVA) the HRWE on the inner shells $J$ and $H$. For these solves inner Dirichlet boundary conditions are the fixed physical ones,

${ }^{6}$ Whereas the basic spectral elements (such as shell $J$, block $B$, and cylinder 1) have been called subdomains, we informally refer to the multidomains $R$ and $G$ (defined later) as a subregions. 


\begin{tabular}{|c|c|c|c|c|c|c|c|c|}
\hline \multicolumn{10}{|c|}{$\Omega=0.1$} \\
\hline \hline$N_{r}$ & $N_{\phi}$ & $N_{z}$ & $L_{2}$ error & $L_{2}$ norm & $L_{\infty}$ error & $L_{\infty}$ norm & iterations & tolerance \\
\hline 13 & 5 & 7 & $7.9884 \mathrm{E}-08$ & $9.0116 \mathrm{E}-01$ & $4.6388 \mathrm{E}-07$ & $1.5004 \mathrm{E}+00$ & 3 & $1.0000 \mathrm{E}-08$ \\
19 & 9 & 9 & $5.2802 \mathrm{E}-10$ & $8.9887 \mathrm{E}-01$ & $2.7463 \mathrm{E}-09$ & $1.5006 \mathrm{E}+00$ & 4 & $1.0000 \mathrm{E}-10$ \\
23 & 13 & 13 & $5.6239 \mathrm{E}-13$ & $8.9775 \mathrm{E}-01$ & $4.4170 \mathrm{E}-12$ & $1.5006 \mathrm{E}+00$ & 5 & $1.0000 \mathrm{E}-12$ \\
29 & 19 & 18 & $8.3992 \mathrm{E}-15$ & $8.9680 \mathrm{E}-01$ & $9.3259 \mathrm{E}-14$ & $1.5007 \mathrm{E}+00$ & 6 & $1.0000 \mathrm{E}-14$ \\
\hline
\end{tabular}

\begin{tabular}{|c|c|c|c|c|c|c|c|c|}
\hline \multicolumn{10}{|c|}{$\Omega=0.3$} \\
\hline \hline$N_{r}$ & $N_{\phi}$ & $N_{z}$ & $L_{2}$ error & $L_{2}$ norm & $L_{\infty}$ error & $L_{\infty}$ norm & iterations & tolerance \\
\hline 13 & 5 & 7 & $6.2980 \mathrm{E}-06$ & $9.4046 \mathrm{E}-01$ & $3.8531 \mathrm{E}-05$ & $1.5817 \mathrm{E}+00$ & 6 & $1.0000 \mathrm{E}-08$ \\
19 & 9 & 9 & $1.2577 \mathrm{E}-07$ & $9.3796 \mathrm{E}-01$ & $5.9139 \mathrm{E}-07$ & $1.5841 \mathrm{E}+00$ & 10 & $1.0000 \mathrm{E}-10$ \\
23 & 13 & 13 & $4.9307 \mathrm{E}-09$ & $9.3677 \mathrm{E}-01$ & $4.0773 \mathrm{E}-08$ & $1.5849 \mathrm{E}+00$ & 14 & $1.0000 \mathrm{E}-12$ \\
29 & 19 & 18 & $3.2422 \mathrm{E}-10$ & $9.3574 \mathrm{E}-01$ & $1.3965 \mathrm{E}-09$ & $1.5861 \mathrm{E}+00$ & 18 & $1.0000 \mathrm{E}-14$ \\
\hline \hline
\end{tabular}

\begin{tabular}{|c|c|c|c|c|c|c|c|c|}
\hline \multicolumn{10}{|c|}{$\Omega=0.5$} \\
\hline \hline$N_{r}$ & $N_{\phi}$ & $N_{z}$ & $L_{2}$ error & $L_{2}$ norm & $L_{\infty}$ error & $L_{\infty}$ norm & iterations & tolerance \\
\hline 13 & 5 & 7 & $7.3178 \mathrm{E}-04$ & $1.0239 \mathrm{E}+00$ & $3.9733 \mathrm{E}-03$ & $1.7886 \mathrm{E}+00$ & 59 & $1.0000 \mathrm{E}-08$ \\
19 & 9 & 9 & $2.1193 \mathrm{E}-04$ & $1.0218 \mathrm{E}+00$ & $1.2227 \mathrm{E}-03$ & $1.8228 \mathrm{E}+00$ & 161 & $1.0000 \mathrm{E}-10$ \\
23 & 13 & 13 & $4.0853 \mathrm{E}-05$ & $1.0203 \mathrm{E}+00$ & $1.8564 \mathrm{E}-04$ & $1.8303 \mathrm{E}+00$ & 531 & $1.0000 \mathrm{E}-12$ \\
29 & 19 & 18 & $7.0259 \mathrm{E}-06$ & $1.0190 \mathrm{E}+00$ & $7.7578 \mathrm{E}-05$ & $1.8285 \mathrm{E}+00$ & 1576 & $1.0000 \mathrm{E}-14$ \\
\hline \hline
\end{tabular}

\begin{tabular}{|c|c|c|c|c|c|c|c|c|}
\hline \multicolumn{10}{|c|}{$\Omega=0.7$} \\
\hline \hline$N_{r}$ & $N_{\phi}$ & $N_{z}$ & $L_{2}$ error & $L_{2}$ norm & $L_{\infty}$ error & $L_{\infty}$ norm & iterations & tolerance \\
\hline 13 & 5 & 7 & $3.2460 \mathrm{E}+00$ & $3.4276 \mathrm{E}+00$ & $2.2390 \mathrm{E}+01$ & $2.1481 \mathrm{E}+01$ & 130 & $1.0000 \mathrm{E}-08$ \\
19 & 9 & 9 & $2.3293 \mathrm{E}-02$ & $1.1548 \mathrm{E}+00$ & $1.9059 \mathrm{E}-01$ & $2.5635 \mathrm{E}+00$ & 503 & $1.0000 \mathrm{E}-10$ \\
23 & 13 & 13 & $1.1546 \mathrm{E}+00$ & $1.6568 \mathrm{E}+00$ & $9.4853 \mathrm{E}+00$ & $1.1688 \mathrm{E}+01$ & 1420 & $1.0000 \mathrm{E}-12$ \\
29 & 19 & 18 & $1.1305 \mathrm{E}-02$ & $1.1512 \mathrm{E}+00$ & $1.1635 \mathrm{E}-01$ & $2.5463 \mathrm{E}+00$ & 10816 & $1.0000 \mathrm{E}-14$ \\
\hline
\end{tabular}

TABLE VIII. CyLindRICAL SHELl 5 TEST With PRECONDitioning.

while outer boundary conditions stem from interpolation of the numerical solution on $R$ (which is initially zero). The tolerance for these solves is typically $0.1 *$ tol, where tol is the tolerance for the global GMRES solve of $\mathcal{M} \widetilde{\Psi}=\mathcal{B \mathcal { G }}$.

2. Solve (also by GMRES) the HRWE on $R$. For this solve inner Dirichlet boundary conditions stem from interpolation of the solutions on $J$ and $H$, while outer Dirichlet boundary conditions stem from interpolation of the solution on the outer shell $O$ (which is initially zero). This GMRES solve must also be preconditioned, as discussed shortly. The tolerance for this solve is typically $0.2 *$ tol.

3. Solve the HRWE on the outer spherical shell $O$, with inner Dirichlet boundary conditions stemming from interpolation of the numerical solution on $R$ and the outer radiation boundary conditions described in Sec. IIA. As described in Sec. IVA, this solve is performed via direct block-by-block $L U$ factorization (note that the factorization of each block mode is precomputed and then used over and over in this third step).

This three-step iteration may be viewed as the Gauss-Seidel method, here applied in block form to $J \cup H, R, O$. Typically, we have chosen 4 sweeps of this block Gauss-Seidel method. Step 2 requires its own preconditioning to enhance convergence. Here we have again employed the alternating Schwarz method, now with blocks corresponding to $B, C$, $D$, and the subregion $G$ which is the composite of glued cylinders (1-5). This "inner" preconditioning 


\begin{tabular}{|c|c|c|c|c|c|c|c|c|}
\hline \multicolumn{10}{|c|}{$\Omega=0.1$} \\
\hline \hline$N_{x}$ & $N_{y}$ & $N_{z}$ & $L_{2}$ error & $L_{2}$ norm & $L_{\infty}$ error & $L_{\infty}$ norm & iterations & tolerance \\
\hline 14 & 14 & 7 & $3.7513 \mathrm{E}-07$ & $1.1367 \mathrm{E}+00$ & $4.2360 \mathrm{E}-06$ & $1.7854 \mathrm{E}+00$ & 41 & $1.0000 \mathrm{E}-08$ \\
19 & 19 & 9 & $6.3235 \mathrm{E}-09$ & $1.1394 \mathrm{E}+00$ & $1.3616 \mathrm{E}-07$ & $1.8098 \mathrm{E}+00$ & 62 & $1.0000 \mathrm{E}-10$ \\
28 & 28 & 13 & $1.4351 \mathrm{E}-11$ & $1.1418 \mathrm{E}+00$ & $3.0822 \mathrm{E}-10$ & $1.8040 \mathrm{E}+00$ & 102 & $1.0000 \mathrm{E}-12$ \\
32 & 32 & 18 & $1.2749 \mathrm{E}-13$ & $1.1421 \mathrm{E}+00$ & $5.3182 \mathrm{E}-12$ & $1.8054 \mathrm{E}+00$ & 141 & $1.0000 \mathrm{E}-14$ \\
\hline
\end{tabular}

\begin{tabular}{|c|c|c|c|c|c|c|c|c|}
\hline \multicolumn{10}{|c|}{$\Omega=0.3$} \\
\hline \hline$N_{x}$ & $N_{y}$ & $N_{z}$ & $L_{2}$ error & $L_{2}$ norm & $L_{\infty}$ error & $L_{\infty}$ norm & iterations & tolerance \\
\hline 14 & 14 & 7 & $3.9020 \mathrm{E}-07$ & $1.1955 \mathrm{E}+00$ & $4.2789 \mathrm{E}-06$ & $1.8911 \mathrm{E}+00$ & 42 & $1.0000 \mathrm{E}-08$ \\
19 & 19 & 9 & $6.4495 \mathrm{E}-09$ & $1.1986 \mathrm{E}+00$ & $1.4194 \mathrm{E}-07$ & $1.9176 \mathrm{E}+00$ & 65 & $1.0000 \mathrm{E}-10$ \\
28 & 28 & 13 & $1.4448 \mathrm{E}-11$ & $1.2013 \mathrm{E}+00$ & $4.9319 \mathrm{E}-10$ & $1.9116 \mathrm{E}+00$ & 109 & $1.0000 \mathrm{E}-12$ \\
32 & 32 & 18 & $8.4807 \mathrm{E}-14$ & $1.2017 \mathrm{E}+00$ & $2.9017 \mathrm{E}-12$ & $1.9131 \mathrm{E}+00$ & 154 & $1.0000 \mathrm{E}-14$ \\
\hline
\end{tabular}

\begin{tabular}{|c|c|c|c|c|c|c|c|c|}
\hline \multicolumn{10}{|c|}{$\Omega=0.5$} \\
\hline \hline$N_{x}$ & $N_{y}$ & $N_{z}$ & $L_{2}$ error & $L_{2}$ norm & $L_{\infty}$ error & $L_{\infty}$ norm & iterations & tolerance \\
\hline 14 & 14 & 7 & $7.5596 \mathrm{E}-07$ & $1.3306 \mathrm{E}+00$ & $4.0594 \mathrm{E}-06$ & $2.1990 \mathrm{E}+00$ & 77 & $1.0000 \mathrm{E}-08$ \\
19 & 19 & 9 & $9.7796 \mathrm{E}-09$ & $1.3351 \mathrm{E}+00$ & $1.6231 \mathrm{E}-07$ & $2.2244 \mathrm{E}+00$ & 266 & $1.0000 \mathrm{E}-10$ \\
28 & 28 & 13 & $2.2103 \mathrm{E}-10$ & $1.3391 \mathrm{E}+00$ & $2.8426 \mathrm{E}-09$ & $2.2217 \mathrm{E}+00$ & 1495 & $1.0000 \mathrm{E}-12$ \\
32 & 32 & 18 & $4.3594 \mathrm{E}-12$ & $1.3399 \mathrm{E}+00$ & $5.1787 \mathrm{E}-11$ & $2.2231 \mathrm{E}+00$ & 3089 & $1.0000 \mathrm{E}-14$ \\
\hline
\end{tabular}

\begin{tabular}{|c|c|c|c|c|c|c|c|c|}
\hline \multicolumn{10}{|c|}{$\Omega=0.7$} \\
\hline \hline$N_{x}$ & $N_{y}$ & $N_{z}$ & $L_{2}$ error & $L_{2}$ norm & $L_{\infty}$ error & $L_{\infty}$ norm & iterations & tolerance \\
\hline 14 & 14 & 7 & $4.0731 \mathrm{E}-03$ & $1.5016 \mathrm{E}+00$ & $1.9997 \mathrm{E}-02$ & $3.0390 \mathrm{E}+00$ & 454 & $1.0000 \mathrm{E}-08$ \\
19 & 19 & 9 & $3.9332 \mathrm{E}-04$ & $1.5065 \mathrm{E}+00$ & $2.1243 \mathrm{E}-03$ & $3.1084 \mathrm{E}+00$ & 1349 & $1.0000 \mathrm{E}-10$ \\
28 & 28 & 13 & $2.3057 \mathrm{E}-06$ & $1.5118 \mathrm{E}+00$ & $1.3776 \mathrm{E}-05$ & $3.1019 \mathrm{E}+00$ & 5337 & $1.0000 \mathrm{E}-12$ \\
32 & 32 & 18 & $1.0375 \mathrm{E}-06$ & $1.5133 \mathrm{E}+00$ & $6.6072 \mathrm{E}-06$ & $3.0967 \mathrm{E}+00$ & $20000^{*}$ & $1.0000 \mathrm{E}-14$ \\
\hline
\end{tabular}

TABLE IX. Block $D$ Test with preconditioning. The asterisk on 20000* indicates the convergence was halted before the tolerance had been achieved; the achieved tolerance $1.3 \times 10^{-14}$ was close to that requested.

typically involves 5 sweeps, with appropriate interpolation. Each individual GMRES solve on $B, C, D$, and $G$ uses the tolerance $0.1 *$ tol. Table $\mathrm{X}$ depicts the overall multilevel preconditioning scheme.

Before turning to tests of the full solve, we consider the solve on the multidomain subregion $G$ comprised of the glued cylinders (1-5). Again, this solve is performed as part of the preconditioner for step 2 of the global preconditioner (see Table X]). Table XI collects errors and iteration counts associated with this solve for increasing truncations. Each solve documented in the table has been started with the zero vector as initial iterate, and here we employ restarting after 20 iterations. The reported iteration counts in Table XI are cumulative over restarts. The individual block- $L U$ preconditioning on each subdomain (1-5) is the only preconditioning used for this solve. Nevertheless, it suffices to drastically reduce the number of iterations (which would otherwise be in the thousands, with or without restarts).

Results for the full solve appear in Table XII. Notice that the largest truncation involves more than half a million unknowns (597788 to be precise). In fact the number of unknowns is larger, since we add modes to shells, but here count only the "physical modes" for allowable $(\ell, m)$ pairs (cf. Sec. IA ). Each solve in the table is used as the initial guess for the next, which is why the count of outer GMRES iterations goes down. 


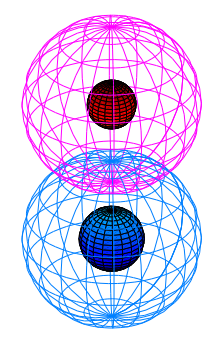

(a) Inner shells $J$ and $H$.

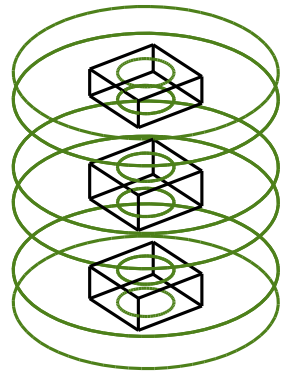

(b) Glued subregion $R$.

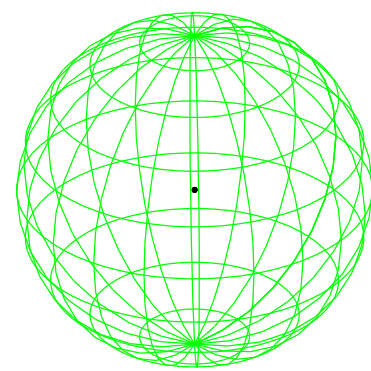

(c) Outer shell $O$.

FIG. 3. Alternating Schwarz Preconditioner. Numerical solution of the HRWE each subdomain/subregion above defines the preconditioner. Boundary conditions for the solves are obtained through subdomain/subregion interpolation as described in the text. For the outer shell shown in (c), the small dot in the center is, to scale, the inner configuration comprised of (a) and (b).

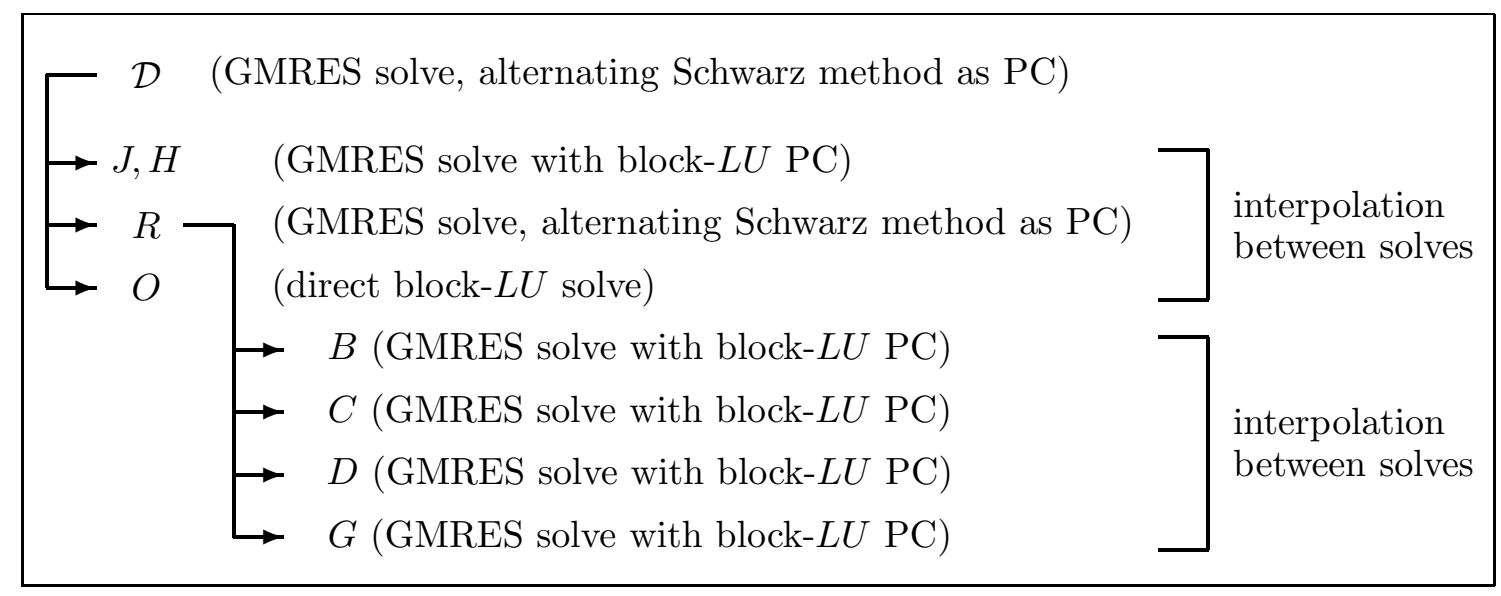

TABLE X. Multilevel PRECONDITIONING SCHEME.

\section{CONCLUSION}

We close by summarizing the results of this paper and describing our outlook on future work. In both the summary and description, we discuss both our numerical methods and the physical problem we aim to solve.

\section{A. Results}

We have demonstrated the feasibility of solving a partial differential equation in three independent variables by modal spectral methods based on the technique of integration preconditioning. As designed, the technique yields an algorithmic way to achieve a sparse 


\begin{tabular}{|c|c|c|c|c|c|c|c|c|c|c|c|c|}
\hline \multicolumn{10}{|c|}{$\Omega=0.1$} \\
\hline \hline$N_{r}^{1}$ & $N_{\phi}^{1}$ & $N_{z}^{1}$ & $N_{z}^{2}$ & $N_{z}^{3}$ & $N_{z}^{4}$ & $N_{z}^{5}$ & $L_{2}$ error & $L_{2}$ norm & $L_{\infty}$ error & $L_{\infty}$ norm & iterations & tolerance \\
\hline 13 & 5 & 7 & 17 & 7 & 17 & 7 & $2.2251 \mathrm{E}-06$ & $9.8806 \mathrm{E}-01$ & $2.7804 \mathrm{E}-05$ & $2.4773 \mathrm{E}+00$ & 17 & $1.0000 \mathrm{E}-06$ \\
19 & 9 & 9 & 23 & 9 & 23 & 9 & $3.5812 \mathrm{E}-08$ & $1.0077 \mathrm{E}+00$ & $2.2023 \mathrm{E}-07$ & $2.4781 \mathrm{E}+00$ & 22 & $1.0000 \mathrm{E}-08$ \\
23 & 13 & 14 & 31 & 16 & 31 & 14 & $1.2344 \mathrm{E}-10$ & $1.0063 \mathrm{E}+00$ & $1.2046 \mathrm{E}-09$ & $2.4782 \mathrm{E}+00$ & 28 & $1.0000 \mathrm{E}-10$ \\
29 & 19 & 15 & 35 & 15 & 35 & 15 & $6.6478 \mathrm{E}-12$ & $1.0083 \mathrm{E}+00$ & $7.4462 \mathrm{E}-11$ & $2.4783 \mathrm{E}+00$ & 42 & $1.0000 \mathrm{E}-12$ \\
29 & 19 & 18 & 39 & 21 & 39 & 18 & $4.9252 \mathrm{E}-13$ & $1.0063 \mathrm{E}+00$ & $5.3570 \mathrm{E}-12$ & $2.4783 \mathrm{E}+00$ & 45 & $1.0000 \mathrm{E}-13$ \\
\hline
\end{tabular}

TABle XI. Solution of the HRWE on the glued Cylinder subregion $G$. The reported truncations $N_{r}^{1}$ and $N_{\phi}^{1}$ were also used for cylinders $2,3,4$, and 5 .

\begin{tabular}{|c|c|c|c|c|c|c|}
\hline \multicolumn{8}{|c|}{$\Omega=0.1$} \\
\hline \hline MPSPD & $L_{2}$ error & $L_{2}$ norm & $L_{\infty}$ error & $L_{\infty}$ norm & iterations & tolerance \\
\hline 15.7 & $3.7532 \mathrm{E}-06$ & $7.0509 \mathrm{E}-01$ & $9.9579 \mathrm{E}-05$ & $3.6556 \mathrm{E}+00$ & 5 & $1.0000 \mathrm{E}-05$ \\
23.9 & $4.2440 \mathrm{E}-08$ & $7.8382 \mathrm{E}-01$ & $5.8222 \mathrm{E}-07$ & $3.6563 \mathrm{E}+00$ & 3 & $1.0000 \mathrm{E}-07$ \\
31.0 & $2.6333 \mathrm{E}-10$ & $8.3492 \mathrm{E}-01$ & $4.0406 \mathrm{E}-09$ & $3.6564 \mathrm{E}+00$ & 3 & $1.0000 \mathrm{E}-09$ \\
37.2 & $4.1855 \mathrm{E}-12$ & $9.3982 \mathrm{E}-01$ & $8.6696 \mathrm{E}-11$ & $3.6565 \mathrm{E}+00$ & 3 & $1.0000 \mathrm{E}-11$ \\
37.9 & $4.7733 \mathrm{E}-13$ & $9.5252 \mathrm{E}-01$ & $8.2254 \mathrm{E}-12$ & $3.6565 \mathrm{E}+00$ & 2 & $1.0000 \mathrm{E}-12$ \\
\hline
\end{tabular}

TABLE XII. Solution of the HRWE on the 2-Center Multidomain $\mathcal{D}$. Here MPSPD stands for modes per subdomain per dimension. Note that an MPSPD of 37.9 corresponds to (11 subdomains $) \times\left(37.9^{3}\right) \simeq 599000$ unknowns.

spectral formulation of the PDE problem with consistent incorporation of boundary conditions. However, particularly in higher dimensional settings, an integration "preconditioner" may not be an optimal approximate inverse in any known sense; as a result the technique would not seem practical in and of itself. Here we mean that, for a higher dimensional problem like ours, the sole use of integration preconditioning will likely lead to prohibitively large iteration counts when using Krylov methods and/or loss of accuracy due to poor conditioning. At least for our problem, we have demonstrated that both issues may be surmounted by further preconditioning. In particular, studying our problem on a given subdomain (spectral element), we have empirically demonstrated that block Jacobi preconditioning (with each block inverted by $L U$ factorization) is effective for the banded matrix produced by integration preconditioning. Moreover, for the matching of subdomains in our multidomain approach the alternating Schwarz method (an elementary domain decomposition preconditioner) works well. Given that little seems known about preconditioning for modal methods, whereas preconditioning for nodal methods is well developed, we believe that our demonstration of effective modal preconditioning based on rather standard methods is remarkable.

In addition to modal preconditioning, other aspects of our work are new from the standpoint of modal spectral methods, in particular its multidomain character and focus on a mixed-type problem. Ref. [1] already presented the outline for applying the integration preconditioning technique to higher dimensional problems, that is to PDEs. While we have carried out and presented the details of such an application, our work has gone further in developing a 3D multidomain version of the technique (Ref. [6] consider the multidomain case in 2D). In particular, we have presented the details of gluing constituent subdomains, and how this gluing is reflected in the overall linear system. As another new, and unusual, aspect, our work is the first successful application of integration preconditioning to a three dimensional mixed-type problem, a problem with both elliptic and hyperbolic regions. Whence it has numerically confirmed once more (cf. [6, 26, 27, 29]) that such problems can 
be well-posed; see [44] for a theoretical discussion. The use of a multidomain decomposition is of special interest for mixed problems like ours, since the type change need not occur in all subdomains. Indeed, for our example, it occurs on a cylinder that intersects only the outer spherical shell. When the nonlinearities of the actual physical problem are included, this feature of our domain decomposition may prove useful, because the true physical equations will be only mildly nonlinear on the outer shell, with the strongest nonlinearities confined to subdomains on which the equations are elliptic. Our work therefore suggests that we might treat the outer shell differently from the inner subdomains when solving the full nonlinear problem.

\section{B. Outlook}

While we have demonstrated that our mix of methods delivers efficiency and remarkable accuracy when applied to a nontrivial 3D model problem, a number of issues merit further investigation. These include both particular challenges in the application of this paper's methods to helically symmetric general relativistic binary fields (the problem of our interest), and numerical analysis questions pertaining to integration preconditioning as a method for more general problems.

The numerical analysis issues center on the value of integration preconditioning, or sparsification, in the solution of higher dimensional PDEs, particularly in the context of a multidomain approach. Here we have applied the method to only one linear PDE, with an empirical demonstration of its success. For any given linear equation, a fuller investigation of integration sparsification for multidomain scenarios would focus on the interplay between condition number, field of values (Rayleigh quotients), and computational efficiency (iteration counts). All of these issues would be examined both before and after some form of "ordinary" preconditioning, e.g. the combination of block- $L U$ and alternating Schwarz preconditioning used in this paper. The sparse matrices produced by integration sparsification allow for quicker matrix multiplies in a Krylov method like GMRES. Our work suggests that this advantage might be gained without large iteration counts, but the issue deserves more careful study. The efficient treatment of nonlinearities is also worthy of investigation, and any such study would build upon the results already given in Ref. [1]. At present, we are in process of evaluating integration sparsification in the context of these issues, mostly with 2D model problems.

Several challenges remain if we are to apply some variant of our method to the problem of helically symmetric general relativistic binary fields. First, we must test the efficiency of our method in solving a nonlinear HRWE. In practice, this should not be a problem. The strongest nonlinearities will occur closest to the black hole sources, i.e. near the surfaces on which the inner boundary conditions are set. By choosing these boundaries some distance from the sources, we can, at the cost of accuracy in mathematically representing the physical problem, reduce the severity of the nonlinearities. The real question, then, is not whether we can handle nonlinearities, but how close to the sources the inner boundaries can be placed. Second, we must replace the outgoing radiative boundary conditions with "standing wave boundary conditions," as described in Ref. [25]. This change is straightforward in a linearized problem, and, as explained in Ref. [25], should not pose great difficulty in nonlinear general relativity. Third, we must move from the scalar problem considered here to the actual tensor problem. Solution of the helically symmetric problem of a binary in full general relativity will require all the information in the tensorial fields, and the coupling of those fields. This 
proved to be the greatest challenge for the solution method presented in Ref. [29], and it severely limited the achievable accuracy. We are confident that the method described in this paper will deliver the accuracy needed to find useful solutions.

The methods developed here have been motivated by the problem of binary inspiral in general relativity. However, our methods may find broader use; they might be applied to problems distinct from the helically symmetric mixed PDEs of the periodic standing wave approximation. As a salient example, multidomain spectral methods are already being used in the elliptical problem of generating binary black hole initial data [30, 31]. Our set of methods, with integration sparsification, might be used as an alternative approach.

\section{ACKNOWLEDGMENTS}

We gratefully acknowledge support from NSF grants PHY 0855678 to the University of New Mexico and PHY 0554367 to the University of Texas at Brownsville. For helpful comments and discussions we thank T. Hagstrom, J. Hesthaven, H. Pfeiffer, G. von Winckel, and particularly E. Coutsias.

\section{Appendix A: Explicit solution for a point source.}

This appendix presents two representations for an exact solution to the HRWE, namely the solution for a point source in a circular orbit. Superposition of two such solutions yields the binary field exploited in our numerical tests. As before, let $(\tilde{x}, \tilde{y}, z=\tilde{z})=$ $(r \sin \theta \cos \varphi, r \sin \theta \sin \varphi, r \cos \theta)$ represent the comoving Cartesian coordinates, where $\varphi=$ $\phi-\Omega t$. In terms of the comoving coordinates, we define the Laplacian $\tilde{\nabla}^{2} \equiv \partial_{\tilde{x}}^{2}+\partial_{\tilde{y}}^{2}+\partial_{z}^{2}$ and $\partial_{\varphi}$ operators and consider the inhomogeneous HRWE

$$
\left(\tilde{\nabla}^{2}-\Omega^{2} \partial_{\varphi}^{2}\right) \psi=-4 \pi \frac{\delta(r-a)}{a^{2}} \delta(\cos \theta) \delta\left(\varphi-\varphi_{0}\right),
$$

where $\left(a, \pi / 2, \varphi_{0}\right)$ specifies the location of the source point in the spherical polar system associated with $(\tilde{x}, \tilde{y}, z)$. We set $\varphi_{0}=0$, since this shift can always be reinserted via the replacement $\varphi \rightarrow \varphi-\varphi_{0}$ in the representations (A2) and (A11) given below. Using standard methods of separation of variables and one-dimensional Green's functions, we find the series representation for a particular solution to (A1),

$$
\begin{aligned}
\psi(\tilde{x}, \tilde{y}, z) & =2 \sum_{\ell=0}^{\infty} \frac{1}{2 \ell+1} \bar{P}_{\ell 0}(\cos \theta) \bar{P}_{\ell 0}(0) \frac{r_{<}^{\ell}}{r_{>}^{\ell+1}} \\
& -4 \Omega \sum_{\ell=1}^{\infty} \sum_{m=1}^{\ell} m \bar{P}_{\ell m}(\cos \theta) \bar{P}_{\ell m}(0) j_{\ell}\left(m \Omega r_{<}\right)\left[n_{\ell}\left(m \Omega r_{>}\right) \cos (m \varphi)+j_{\ell}\left(m \Omega r_{>}\right) \sin (m \varphi)\right] .
\end{aligned}
$$

Here $\bar{P}_{\ell m}(u)$ is a normalized associated Legendre function, and $j_{\ell}(z)$ and $n_{\ell}(z)$ are respectively spherical Bessel functions of the first and second kind [38]. Moreover, we adopt the standard notations $r_{<}=\min (a, r)$ and $r_{>}=\max (a, r)$. The series is poorly convergent near $r=a$; however, for $\Omega \ll 1$ it converges rapidly for $r \gg a$. 
To derive a separate representation of the same series which can be used near $r=a$, we consider the equivalent problem for the $3+1$ wave equation written in inertial, rather than comoving coordinates,

$$
\left(\nabla^{2}-\partial_{t}^{2}\right) \Psi=-4 \pi \frac{\delta(r-a)}{a^{2}} \delta(\cos \theta) \delta(\phi-\Omega t) .
$$

In the inertial frame the source point has the time-dependent location

$$
\boldsymbol{\xi}(t)=a \cos (\Omega t) \mathbf{e}_{x}+a \sin (\Omega t) \mathbf{e}_{y} .
$$

Therefore, we wish to find the retarded solution to

$$
\left(\nabla^{2}-\partial_{t}^{2}\right) \Psi=-4 \pi \delta^{(3)}(\mathbf{x}-\boldsymbol{\xi}(t)),
$$

and then evaluate it at the field point

$$
\mathbf{x}(t)=z \mathbf{e}_{z}+\rho \cos (\phi+\Omega t) \mathbf{e}_{x}+\rho \sin (\phi+\Omega t) \mathbf{e}_{y},
$$

where $\rho^{2}=x^{2}+y^{2}=\tilde{x}^{2}+\tilde{y}^{2}$. Notice that the evaluation point $\mathbf{x}(t)$ rotates with the source; whence this latter evaluation will effectively remove the time dependence. The retarded-time Green's function for the wave operator is

$$
G_{\mathrm{ret}}\left(t, \mathbf{x} ; t^{\prime}, \mathbf{x}^{\prime}\right)=\frac{1}{4 \pi} \frac{\delta\left(t-t^{\prime}-\left|\mathbf{x}-\mathbf{x}^{\prime}\right|\right)}{\left|\mathbf{x}-\mathbf{x}^{\prime}\right|}
$$

and it obeys

$$
\left(\nabla^{2}-\partial_{t}^{2}\right) G_{\text {ret }}\left(t, \mathbf{x} ; t^{\prime}, \mathbf{x}^{\prime}\right)=-\delta\left(t-t^{\prime}\right) \delta^{(3)}\left(\mathbf{x}-\mathbf{x}^{\prime}\right) .
$$

We obtain the desired solution to (A5) via spacetime convolution of $4 \pi \delta^{(3)}(\boldsymbol{\xi}(t))$ with $G_{\text {ret }}\left(t, \mathbf{x} ; t^{\prime}, \mathbf{x}^{\prime}\right)$. The details of this calculation are given in the textbook by Matthews and Walker [43], and the result is

$$
\Psi(t, \mathbf{x})=\frac{1}{\left|\mathbf{x}-\boldsymbol{\xi}\left(t^{\prime}\right)\right|-\dot{\boldsymbol{\xi}}\left(t^{\prime}\right) \cdot\left(\mathbf{x}-\boldsymbol{\xi}\left(t^{\prime}\right)\right)},
$$

where $\dot{\boldsymbol{\xi}}$ denotes the derivative of $\boldsymbol{\xi}\left(t^{\prime}\right)$ with respect to its argument. Here $t^{\prime}$ is retarded time which obeys $t-t^{\prime}=\left|\mathbf{x}-\boldsymbol{\xi}\left(t^{\prime}\right)\right|$. Setting $\mathbf{x}=\mathbf{x}(t)$, we find

$$
\begin{aligned}
\left(t-t^{\prime}\right) & =\left[z^{2}+\rho^{2}+a^{2}-2 a \rho \cos \left(\phi-\Omega t^{\prime}\right)\right]^{1 / 2} \\
& =\left[z^{2}+\rho^{2}+a^{2}-2 a \rho \cos \left(\varphi+\Omega\left(t-t^{\prime}\right)\right)\right]^{1 / 2} .
\end{aligned}
$$

With $(\rho, z, \varphi)$ near $(a, 0,0)$, we may use the last formula to numerically compute $t-t^{\prime}$ via fixed-point iteration. Finally, since $\psi(\tilde{x}, \tilde{y}, z)=\Psi(t, \mathbf{x}(t))$, we then have

$$
\psi(\tilde{x}, \tilde{y}, z)=\frac{1}{\left[z^{2}+\rho^{2}+a^{2}-2 a \rho \cos \left(\varphi+\Omega\left(t-t^{\prime}\right)\right)\right]^{1 / 2}-a \rho \Omega \sin \left(\varphi+\Omega\left(t-t^{\prime}\right)\right)}
$$


as a concrete expression for (A9). With both Eqs. (A2) and (A11) at our disposal, we can evaluate the retarded solution to (A1) with enough accuracy (uniformly over the entire 2-center domain) to make the comparisons reported in Sec. IV.

[1] E. A. Coutsias, T. Hagstrom, J. S. Hesthaven, and D. Torres, Integration preconditioners for differential operators in spectral $\tau$-methods, Spec. Issue Houston Journal of Mathematics, 21 (1996).

[2] E. A. Coutsias, T. Hagstrom, and D. Torres, An efficient spectral method for ordinary differential equations with rational function coefficients, Math. Comput., vol. 65, no. 214, pp. 611-635 (1996).

[3] J. S. Hesthaven, Integration preconditioning of pseudospectral operators. I. Basic linear operators, SIAM J. Numer. Anal., vol. 35, no. 4, pp. 1571-1593 (1998).

[4] D. J. Torres and E. A. Coutsias, Pseudospectral solution of the two-dimensional Navier-Stokes equations in a disk, SIAM J. Sci. Comput., vol. 21, no. 1, pp. 378-403 (1999).

[5] G. von Winckel, S. Krishna, and E. A. Coutsias, Spectral element modeling of semiconductor heterostructures, Mathematical and Computer Modeling, vol. 43, issues 5-6, pp. 582-591 (2006).

[6] S. R. Lau and R. H. Price, Multidomain Spectral Method for the Helically Reduced Wave Equation, J. Comput. Phys., vol. 227, issue 2, pp. 1126-1161 (2007).

[7] F. Pretorius, Evolution of Binary Black-Hole Spacetimes, Phys. Rev. Lett. 95, 121101 (2005) [4 pages].

[8] M. Campanelli, C. O. Lousto, P. Marronetti, and Y. Zlochower, Accurate Evolutions of Orbiting Black-Hole Binaries without Excision, Phys. Rev. Lett. 96, 111101 (2006) [4 pages].

[9] J. G. Baker, J. Centrella, D.-I. Choi, M. Koppitz, and J. van Meter, Gravitational-Wave Extraction from an Inspiraling Configuration of Merging Black Holes, Phys. Rev. Lett. 96, 111102 (2006) [4 pages].

[10] M. Campanelli, C. O. Lousto, and Y. Zlochower, Spinning-black-hole binaries: The orbital hang-up, Phys. Rev. D 74, 041501(R) (2006) [5 pages].

[11] M. A. Scheel, H. P. Pfeiffer, L. Lindblom, L. E. Kidder, O. Rinne, and S. A. Teukolsky, Solving Einstein's equations with dual coordinate frames, Phys. Rev. D 74, 104006 (2006) [13 pages].

[12] M. Campanelli, C. O. Lousto, Y. Zlochower, B. Krishnan, and D. Merritt, Spin flips and precession in black-hole-binary mergers, Phys. Rev. D 75, 064030 (2007) [17 pages].

[13] M. Boyle, D. A. Brown, L. E. Kidder, A. H. Mroué, H. P. Pfeiffer, M. A. Scheel, G. B. Cook, and S. A. Teukolsky, High-accuracy comparison of numerical relativity simulations with postNewtonian expansions, Phys. Rev. D, 76 (2007), 124038 [31 pages].

[14] B. Brügmann, J. A. González, M. Hannam, S. Husa, and U. Sperhake, Calibration of moving puncture simulations, Phys. Rev. D 77, 024027 (2008) [25 pages].

[15] M. Campanelli, C. O. Lousto, and Y. Zlochower, Close encounters of three black holes, Phys. Rev. D 77, 101501(R) (2008) [5 pages].

[16] J. G. Baker, W. D. Boggs, J. Centrella, B. J. Kelly, S. T. McWilliams, M. C.Miller, J. R. van Meter, Modeling Kicks from the Merger of Generic Black Hole Binaries, Astrophys. J., vol. 682, no. 1, pp. L29-L32 (2008).

[17] J. G. Baker, W. D. Boggs, J. Centrella, B. J. Kelly, S. T. McWilliams, J. R. van Meter, Mergers of nonspinning black-hole binaries: Gravitational radiation characteristics, Phys. Rev. D 78, 
044046 (2008) [25 pages].

[18] L. Rezzolla, E. N. Dorband, C. Reisswig, P. Diener, D. Pollney, E. Schnetter, and B. Szilágyi, Spin Diagrams for Equal-Mass Black Hole Binaries with Aligned Spins, Astrophys. J., vol. 679, no. 2, pp. 1422-1426 (2008).

[19] M. Campanelli, C. O. Lousto, H. Nakano, and Y. Zlochower, Comparison of numerical and post-Newtonian waveforms for generic precessing black-hole binaries, Phys. Rev. D 79, 084010 (2009) [24 pages].

[20] B. Szilágyi, L. Lindblom, and M. A. Scheel, Simulations of binary black hole mergers using spectral methods, Phys. Rev. D 80, 124010 (2009) [17 pages].

[21] J. A. González, U. Sperhake, and B. Brügmann, Black-hole binary simulations: The mass ratio 101, Phys. Rev. D 79, 124006 (2009) [10 pages].

[22] C. O. Lousto and Y. Zlochower, Orbital Evolution of Extreme-Mass-Ratio Black-Hole Binaries with Numerical Relativity, Phys. Rev. Lett. 106, 041101 (2011) [4 pages].

[23] G. Lovelace, M. A. Scheel, B. Szilágyi, Simulating merging binary black holes with nearly extremal spins, Phys. Rev. D 83, 024010 (2011) [5 pages].

[24] J. Centrella, J. G. Baker, B. J. Kelly, and J. R. van Meter, Black-hole binaries, gravitational waves, and numerical relativity, Rev. Mod. Phys. 82, pp. 3069-3119 (2010).

[25] Z. Andrade, C. Beetle, A. Blinov, B. Bromley, L. M. Burko, M. Cranor, R. Owen, and R. H. Price, The Periodic Standing-Wave Approximation: Overview and Three Dimensional Scalar Models, Phys. Rev. D 70, 064001, (2004) [14 pages].

[26] B. Bromley, R. Owen, and R. H. Price, Periodic standing-wave approximation: Nonlinear scalar fields, adapted coordinates, and the eigenspectral method, Phys. Rev. D 71, 104017 (2005) [27 pages].

[27] C. Beetle, B. Bromley, and R. H. Price, Periodic standing-wave approximation: Eigenspectral computations for linear gravity and nonlinear toy models, Phys. Rev. D 74, 024013 (2006) [17 pages].

[28] C. Beetle, B. Bromley, and R. H. Price, Periodic standing-wave approximation: PostMinkowski computations, Phys. Rev. D 76, 084016 (2007) [16 pages].

[29] N. Hernández and R. H. Price, Periodic standing-wave approximation: Computations in full general relativity, Phys. Rev. D 79, 064008 (2009) [13 pages].

[30] H. Pfeiffer, Initial data for black hole evolutions, Ph.D. thesis, Cornell University, 2003. Available as arXiv:gr-qc/0510016v1.

[31] H. P. Pfeiffer, L. E. Kidder, M. A. Scheel, and S. A. Teukolsky, A multidomain spectral method for solving elliptic equations, Comput. Phys. Commun., vol. 152, issue 3, pp. 253-273 (2003).

[32] Spectral Einstein Code, www.black-holes.org/SpEC.html.

[33] S. A. Orszag, Spectral methods for problems in complex geometries, J. Comput. Phys., vol. 37, issue 1, pp. 70-92 (1980).

[34] S. D. Kim and S. V. Parter, Preconditioning Chebyshev spectral collocation operators by finitedifference operators, SIAM J. Numer. Anal, vol. 34, no. 3, pp. 939-958 (1997).

[35] V. Frayssé, L. Giraud, S. Gratton, and J. Langou, A Set of GMRES Routines for Real and Complex Arithmetics on High Performance Computers, CERFACS Technical Report TR/PA/03/3, July 2007. Available at http://www.cerfacs.fr/algor/.

[36] G. H. Golub and C. F. Van Loan, Matrix Computations (John Hopkins University Press, Baltimore, 1996).

[37] J. Hesthaven, S. Gottlieb, and D. Gottlieb, Spectral Method for Time-Dependent Problems Cambridge University Press, Cambridge, UK, 2007). 
[38] M. Abramowitz and I. Stegun, Handbook of Mathematical Functions (Dover, New York, 1972).

[39] I. J. Thompson and A. R. Barnett, Coulomb and Bessel functions of complex arguments and order, J. Comput. Phys., vol. 64, issue 2, pp. 490-509 (1986).

[40] E. L. Hill and R. Landshoff, The Dirac Electron Theory, Rev. Mod. Phys. 10, pp. 87-132 (1938).

[41] K. S. Thorne, Multipole expansions of gravitational radiation, Rev. Mod. Phys. 52, pp. 299-339 (1980).

[42] B. Smith, P. Bjørstad, and W. Gropp, Domain Decomposition: Parallel Multilevel Methods for Elliptic Partial Differential Equations (Cambridge University Press, Cambridge, UK, 1996).

[43] J. Mathews and R. L. Walker, Mathematical Methods of Physics (W. A. Benjamin, Inc., New York, 1965).

[44] J. Bičák and B. G. Schmidt, Helical symmetry in linear systems, Phys. Rev. D 76, 104040 (2007) [11 pages]. 